\title{
Ferulic Acid Ameliorates Alzheimer's Disease-like Pathology and Repairs Cognitive Decline by Preventing Capillary Hypofunction in APP/PS1 Mice
}

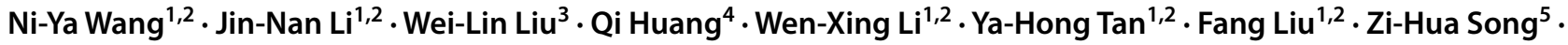 \\ Meng-Yue Wang ${ }^{6} \cdot$ Ning Xie $^{6} \cdot$ Rong-Rong Mao $^{1,7} \cdot$ Ping Gan ${ }^{1,7} \cdot$ Yu-Qiang Ding ${ }^{8} \cdot$ Zhi Zhang $^{5} \cdot$ Bao-Ci Shan ${ }^{4}$.

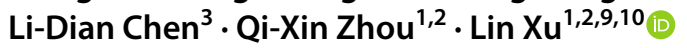

Accepted: 4 February 2021 / Published online: 30 March 2021

(c) The Author(s) 2021

\begin{abstract}
Brain capillaries are crucial for cognitive functions by supplying oxygen and other nutrients to and removing metabolic wastes from the brain. Recent studies have demonstrated that constriction of brain capillaries is triggered by beta-amyloid $(\mathrm{A} \beta)$ oligomers via endothelin-1 (ET1)-mediated action on the ET1 receptor A (ETRA), potentially exacerbating A $\beta$ plaque deposition, the primary pathophysiology of Alzheimer's disease (AD). However, direct evidence is still lacking whether changes in brain capillaries are causally involved in the pathophysiology of AD. Using APP/PS1 mouse model of AD (AD mice) relative to age-matched negative littermates, we identified that reductions of density and diameter of hippocampal capillaries occurred from 4 to 7 months old while $A \beta$ plaque deposition and spatial memory deficit developed at 7 months old. Notably, the injection of ET1 into the hippocampus induced early A $\beta$ plaque deposition at 5 months old in AD mice. Conversely, treatment of ferulic acid against the ETRA to counteract the ET1-mediated vasoconstriction for 30 days prevented reductions of density and diameter of hippocampal capillaries as well as ameliorated $A \beta$ plaque deposition and spatial memory deficit at 7 months old in $\mathrm{AD}$ mice. Thus, these data suggest that reductions of density and diameter of hippocampal capillaries are crucial for initiating $\mathrm{A} \beta$ plaque deposition and spatial memory deficit at the early stages, implicating the development of new therapies for halting or curing memory decline in $\mathrm{AD}$.
\end{abstract}

Key Words Alzheimer's disease $\cdot$ APP/PS1 mouse $\cdot$ A $\beta$ plaque $\cdot$ Hippocampus $\cdot$ Endothelin- $1 \cdot$ Ferulic acid (FA)

\section{Introduction}

As the population ages, Alzheimer's disease (AD) has led to a huge and increasing socioeconomic burden on societies worldwide [1]. Previous studies have established that $\mathrm{A} \beta$

Ni-Ya Wang, Jin-Nan Li, Wei-Lin Liu, Qi Huang, Wen-Xing Li contribute to this work equally.

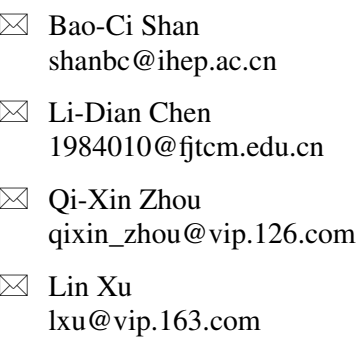

Extended author information available on the last page of the article plaque deposition is the primary pathophysiology of $\mathrm{AD}$ [2-10], but the $A \beta$-directed therapies have failed in halting or curing the progressive decline of memory and other cognitive functions in $\mathrm{AD}$ patients [11]. This arises a critical question of which mechanisms of action relevant to aging could work for the therapy.

Aging is the primary factor associated with vascular changes such as reduced cerebral blood flow (CBF) and particularly correlated with the prevalence of AD [1]. Previous studies have demonstrated that reduced $\mathrm{CBF}$ or brain hypoperfusion is correlated with memory decline in AD [12-20]. Relatively, less attention has been paid to the changes in brain capillaries in AD patients [21,22] or rodent models $[23,24]$. A recent study has further identified an overall reduction of capillary diameter in the hippocampus by using a high-resolution mapping technique [25] in aged APP/PS1 mice. Furthermore, the soluble $A \beta$ oligomers cause capillary constriction via the ET1-mediated ETRA activation [26], 
leading to brain hypoperfusion $[27,28]$. Since brain hypoperfusion likely increases the accumulation and/or decreases the clearance of $A \beta$ [29-33], this could form a feedforward cycle of the hypoperfusion-A $\beta$ aggregation-more hypoperfusion to initiate $A \beta$ plaque deposition. Thus, we assumed that reduced density and/or diameter of the capillaries in the hippocampus could be the earliest events relevant to aging and also for the pathophysiology of AD because cognitive functions in early $\mathrm{AD}$ are mostly restricted to the impairment of episodic memories [34], for which the hippocampus is well known to play a crucial role [35].

However, direct evidence for a causal involvement of hippocampal capillaries in the pathophysiology of AD remains still lacking [36]. To address this question, we used $\mathrm{AD}$ mice and age-matched negative littermates (WT mice) from 3 to 12 months old. We found that reduced density and diameter of hippocampal capillaries were occurring from 4 to 7 months old, but $\mathrm{A} \beta$ plaque deposition, spatial memory deficit, and aggregative microglial cells were all present at 7 months old in AD mice. The injection of ET1 into the hippocampus as a proof of concept for hippocampal hypoperfusion caused early $A \beta$ plaque deposition at 5 months old in $\mathrm{AD}$ mice. In contrast, $\mathrm{FA}$ is a natural compound able to produce antioxidant and anti-inflammation actions [37] or inhibition of $A \beta$ aggregation [38]. We found that FA could bind on the ETRA to counteract ET1-mediated vasoconstriction, and the treatment of FA for 30 days prevented reductions of density and diameter of hippocampal capillaries as well as ameliorated $\mathrm{A} \beta$ plaque deposition, increased aggregative microglial cells, and spatial memory deficit. Thus, we conclude that the changes of hippocampal capillaries are probably crucial for initiating $\mathrm{A} \beta$ plaque deposition and spatial memory deficit at the early stages, implicating the development of new therapies for memory decline in early AD.

\section{Materials and Methods}

\section{Animals}

The male APP/PS1 transgenic mice and their negative littermates [B6. Cg-Tag (APPswe, PSEN1dE9) 85Dbo/ Mmjax, from the Model Animal Research Centre of Nanjing University, Nanjing, China], and C57 mice (from Animal Center of Kunming Medical University, Yunnan, China) were used. Animals were group-housed (4-5 littermates) in ventilated cages with free access to water and food, a 12/12-h light/dark cycle, and a thermoregulated environment. The age and number $(\mathrm{N})$ of these animals were described in the "Results" sections while $n$ represented the number of measured sections or slices. All experimental procedures were approved by the
Institutional Animal Care and Use Committee of Kunming Institute of Zoology, the Chinese Academy of Sciences, Kunming 650223, China (SMKX-2015019).

\section{Ferulic Acid Treatment}

Some AD mice at 6 months old were subjected to ferulic acid (FA) (Aladdin, Shanghai, China) treatment for 30 days via their daily drinking water from the water bottles placed on homecage (4-5 mice/cage). We measured the body weight of each mouse and got the average daily water intake per cage for 3 days before drug administration. In our experimental conditions, each mouse drank about $3.5-5 \mathrm{~mL}$ per day. Then, we prepared the drinking water with FA according to the body weight and the average water intake per day. After starting FA administration, the water bottle was changed twice a week when the drinking amount of each cage was measured. Based on these parameters, the average amount of FA consumption for each mouse was estimated to be about $20 \mathrm{mg} / \mathrm{kg} /$ day. The vehicle control in AD or WT mice at the same age took the normal drinking water without FA.

\section{The Morris Water Maze}

The water maze consists of a 120-cm-diameter circular white plastic tank (Med Association, USA) filled with warm water $\left(21-22^{\circ} \mathrm{C}\right)$, and the polyethylene plastic particles are used to obscure a hidden platform $(10 \mathrm{~cm}$ in diameter) underwater. The experimental procedures used here are like those previously described [39-42]. Briefly, the Morris water maze test includes spatial learning for consecutive 5 days and then $24 \mathrm{~h}$ later a probe trial for testing spatial memory. In the spatial learning task, each animal was gently released into the water maze by facing the wall of the water tank, from four different quadrants in four training trials per day, with a 10-min intertrial interval, and allowed to find the hidden platform in $60 \mathrm{~s}$. If a mouse found the hidden platform within $60 \mathrm{~s}$, it allowed staying on the platform for $10 \mathrm{~s}$. If a mouse failed to escape to the hidden platform in $60 \mathrm{~s}$, it was guided on to and stayed on the platform for $15 \mathrm{~s}$. The mean time in escaping onto the hidden platform was used to score the spatial learning. The probe trial $24 \mathrm{~h}$ after the final training without the hidden platform was used to test spatial memory. In this probe trial, each mouse was released into the water maze also by facing the wall of the water tank, from the diagonal quadrant of the original hidden platform location, and allowed a free swim for $60 \mathrm{~s}$. The time spent in the target quadrant where the hidden platform was previously located was used to score spatial memory. All the data in the Morris water maze test were recorded and analyzed by using the EnthoVision 8.0 program (from Noldus, Beijing, China). 


\section{Immunohistochemistry}

The techniques used here were like those described previously $[43,44]$. Mice were anesthetized with pentobarbital sodium $(80 \mathrm{mg} / \mathrm{kg}$, i.p.) and transcardially perfused with phosphate-buffered saline (PBS) followed by $4 \%$ paraformaldehyde (PFA). Then, the brains were harvested and post-fixed in $4 \%$ PFA at $4{ }^{\circ} \mathrm{C}$ overnight and dehydrated in $30 \%$ sucrose with PBS at $4{ }^{\circ} \mathrm{C}$ for $48 \mathrm{~h}$. The brains were sectioned (coronal sections in $30 \mu \mathrm{m}$ thickness) by using a vibratome (Leica Biosystems, German). The brain sections were stained with antibodies. Besides, for capillary staining (Collagen IV), mice were only transcardially perfused with PBS, and then post-fixed in $4 \%$ PFA at $4{ }^{\circ} \mathrm{C}$ for $8 \mathrm{~h}$, but the other steps were the same.

For immunofluorescence, we used the free-floating immunohistochemistry method. The sections were first washed three times and then blocked in a $0.01 \mathrm{M}$ PBS containing 5\% BSA and $0.3 \%$ Triton $\mathrm{X}-100$ for $1 \mathrm{~h}$ and followed the incubation with the primary antibody overnight at $4{ }^{\circ} \mathrm{C}$. The antibodies used were as follows: anti-A $\beta$ (6E10, 1:1000, sig39300, Covance), (D54D2, 1:1000, 51374, Cell Signaling), Iba1 (1:1000, 019-19741, Wako), GFAP (1:1000, ab53554, Abcam), and collagen IV (1:500, ab6586, Abcam). Next, the slices were washed three times in PBS and followed by 2-h incubation with the fluorescent secondary antibody (1:1000, Life Technologies) at room temperature. Finally, these slices were washed three times and then followed by mounting with 4',6-diamidino-2-phenylindole (DAPI) staining, and cover-slipping on the microscope slides. Images were acquired by using a confocal microscope (Nikon A1 or Olympus FV3000).

DAB (diaminobenzidine) immunostaining was performed on 30- $\mu \mathrm{m}$ sections. The sections were placed into $0.3 \%$ hydrogen peroxide to inhibit endogenous peroxidase for $10 \mathrm{~min}$, and then washed three times and blocked in a 0.01 M PBS containing 5\% BSA and 0.3\% Triton X-100 for $1 \mathrm{~h}$ and followed the incubation with the primary antibody Iba1 (1:1000, 019-19741, Wako) overnight at $4{ }^{\circ} \mathrm{C}$. Next, the sections were washed three times in PBS and followed by $1 \mathrm{~h}$ incubation with biotinylated secondary antibody (1:1000, A0279, Beyotime Biotech) and 30-min incubation with Streptavidin/HRP (1:250, SE068, Solarbio) at room temperature. DAB Horseradish Peroxidase Color Development Kit (P0202, Beyotime Biotech) was used for immunohistochemical color development. Images were acquired by using an optical microscope (Olympus).

Quantification of the confocal images was performed as follows: images were acquired throughout the hippocampus and the cortex from three to six nonadjacent sections $(\sim 180 \mu \mathrm{m}$ apart) per animal. The images were analyzed using the ImageJ software (NIH). The "analyze particles" function was used for the positive area measurement, and a set threshold was used for both control and experimental groups. For capillary diameter measurement, a line was drawn in ImageJ across the capillary perpendicular to its axis and the diameter of the capillary was measured. We measured 3 points per capillary and 10 capillaries per section for each animal.

\section{Thioflavin S Staining}

Thioflavin $S$ (Ths) staining was used to label the $A \beta$ plaques. Briefly, the brain sections were washed three times and then stained with Ths $(0.05 \mathrm{mg} / \mathrm{mL})$ (T1892, Sigma-Aldrich) in dark for 10 min and followed by two washes with 50\% ethanol and PBS. The sections were mounted and imaged by using the confocal microscope.

\section{Surgery and ET1 Injection}

Using the techniques like those described previously [45, 46], the surgery was carried out under pentobarbital sodium $(80 \mathrm{mg} / \mathrm{kg}$, i.p, Sigma-Aldrich) anesthesia, the body temperature $\left(37 \pm 0.5^{\circ} \mathrm{C}\right)$ was maintained through a heating pad, and the scalp was shaved and sterilized with povidoneiodine and 70\% ethanol. Vehicle or ET1 was injected into the hippocampal CA1 region by using a stereotaxic apparatus (RWD Life Sciences, China). Under the surgery conditions, glass micropipettes were positioned to the targeted area by using the stereotaxic coordinates: dorsal CA1 regions in the hippocampus (AP, $-2.0 \mathrm{~mm}, \mathrm{ML} \pm 1.5 \mathrm{~mm}, \mathrm{DV}-1.7 \mathrm{~mm}$ ). Infusion of vehicle or ET1 $(1 \mu \mathrm{L}, 1 \mu \mathrm{g} / \mu \mathrm{L}$, E7764, SigmaAldrich) by using glass micropipettes was driven by a syringe pump (Micro 4, USA) at a speed of $0.1 \mu \mathrm{L} / \mathrm{min}$. These animals were housed in an isolated room for recovery and experiment.

\section{Lectin Perfusion}

After being anesthetized with pentobarbital sodium $(80 \mathrm{mg} /$ $\mathrm{kg}$, i.p., Sigma-Aldrich), the mice were injected with fluorophore-conjugated tomato lectin (DL-1177, Vector Laboratories) $(0.5 \mathrm{mg} / \mathrm{mL}, 100 \mu \mathrm{L})$ via intracardiac. Then the animals were perfused, and the brain was removed and sectioned for microscope imaging.

\section{Ferulic Acid-Biotin Injection and Immunostaining}

Ferulic acid-biotin (FA-biotin) was synthesized by the Shureli Biopharma company (Kunming, China). FA-biotin $(1 \mu \mathrm{L}, 10 \mu \mathrm{g} / \mu \mathrm{L})$ and biotin $(1 \mu \mathrm{L}, 5 \mu \mathrm{g} / \mu \mathrm{L}$, Sigma-Aldrich) were injected into the hippocampus at a speed of $0.1 \mu \mathrm{L} / \mathrm{min}$ as described in the above. After the injection for $40 \mathrm{~min}$ for allowing the drug to be fully diffused, the mice were sacrificed for immunostaining. As described above, the sections 
were incubated with the primary antibody overnight at $4{ }^{\circ} \mathrm{C}$. The antibody used was anti-ETRA (1:800, E9780, SigmaAldrich). Next, the slices were washed three times in PBS and followed by 2-h incubation with the secondary antibody (1:1000, Life Technologies) and Streptavidin-Alexa Fluor ${ }^{\mathrm{TM}}$ 488 conjugate $(1: 1000, \mathrm{~S} 11223$, Invitrogen) at room temperature. Finally, these slices were washed three times with PBS, followed by mounting with DAPI and cover-slipping on the microscope slides. Images were acquired by using a confocal microscope (Nikon A1 or Olympus FV3000).

\section{Enzyme-Linked Immunosorbent and $\beta$-Secretase Activity Assays}

The hippocampus samples were homogenized in ice-cold RIPA Lysis Buffer (Beyotime Biotech), supplemented with the PMSF, protease, and phosphatase inhibitor. The concentrations of $A \beta 1-40$ and $A \beta 1-42$ in the brain extracts were measured by using an enzyme-linked immunosorbent assay (ELISA) according to the manufacturer's instructions (R\&D Systems, DAB142, DAB140B).

The $\beta$-site of the APP Cleaving Enzyme 1 (BACE1) activity in the fresh brain tissues was measured by using the $\beta$-Secretase Activity Fluorometric Assay Kit, according to the manufacturer's instruction (Biovision, K360-100).

\section{Western Blot}

The paradigm used was like those described previously $[40,47]$. The hippocampal tissues of the mice were homogenized in ice-cold RIPA Lysis Buffer (Beyotime Biotech) supplemented with the PMSF (Selleck) and protease inhibitor (Millipore). The homogenates were centrifuged at $1000 \mathrm{~g}$ for $15 \mathrm{~min}$ at $4{ }^{\circ} \mathrm{C}$. Twenty $\mu \mathrm{L}$ of each sample being stored for BCA assay and the rest was mixed with $4 \times$ SDS loading buffer $(250 \mathrm{mmol}$ Tris-HCl, $\mathrm{pH} 6.8,20 \%$ $\beta$-mercaptoethanol, 4\% SDS, $0.004 \%$ bromophenol blue (wt/ $\mathrm{vol}), 40 \%$ (vol/vol) glycerol) in a $3: 1$ ratio, heated at $80{ }^{\circ} \mathrm{C}$ for $15 \mathrm{~min}$. Each sample was run on an SDS-PAGE (10\% acrylamide) and transferred to a PVDF membrane (Millipore). The membranes were blocked for $1 \mathrm{~h}$ with TBST (0.9\% NaCl, $10 \mathrm{mM}$ Tris, $0.1 \%$ Tween-20, PH7.4) containing $5 \% \mathrm{BSA}$ on an orbital shaker at room temperature, and then incubated the primary antibody overnight at $4{ }^{\circ} \mathrm{C}$ (antiamyloid precursor protein, 1:5000, A8717, Sigma-Aldrich; anti-tubulin, 1:10,000, CWbiotech). After three washes for 10 min each with TBST, the membranes were subsequently incubated with HRP-linked secondary antibody (goat $\times$ rabbit/mouse, HRP-linked, 1:10,000, KangChen Biotech Inc, China) for $2 \mathrm{~h}$ at room temperature. Immunoreactivity was detected by using Gel Imaging System (Tannon 5200 Multi) and analyzed by using the Image $\mathbf{J}$ software.

\section{Transmission Electron Microscope}

The method is like a previous study [48]. The slices of the hippocampus $\left(0.1 \mathrm{~mm}^{2}\right)$ were collected from the hippocampus of WT and AD mice. After first being fixed with $3 \%(\mathrm{w} / \mathrm{v})$ glutaraldehyde and $2 \%(\mathrm{w} / \mathrm{v})$ paraformaldehyde, and then stained with $1.5 \%(\mathrm{w} / \mathrm{v})$ potassium ferrocyanide (Sigma-Aldrich) and 1\% (w/v) osmium tetroxide (Ted Pella) in cacodylate buffer $(0.1 \mathrm{M}, \mathrm{pH} 7.4)$ for $40 \mathrm{~min}$, followed by $1 \%(\mathrm{w} / \mathrm{v})$ osmium tetroxide and $2 \%(\mathrm{w} / \mathrm{v})$ uranyl acetate (SPI supplies). The tissues were dehydrated with gradient mixtures of ethanol and acetone, infiltrated by Spi-Pon 812 resin (SPI supplies). The sections with 70-nm thickness were obtained in an ultramicrotome (Leica EM UC7) and then contrasted with uranyl acetate and lead nitrate (Alfa Aesar). Finally, the tissues were detected under a Tecnai $120 \mathrm{kV}$ transmission electron microscope (Thermo Fisher).

\section{Laser Speckle Contrast Imaging}

The cerebral blood flow (CBF) was evaluated by using a laser speckle contrast imaging system (LSCI) [49] (RWD Life Sciences, China). Mice were anesthetized with a mixed air containing $1 \%$ isoflurane through a mask, and placed in a stereotaxic frame (RWD Life Sciences, China). The body temperature $\left(37 \pm 0.5{ }^{\circ} \mathrm{C}\right)$ was maintained through a heating pad (RWD Life Sciences, China). The scalp was then shaved and sterilized with povidone-iodine and 70\% ethanol, and then incised the scalp to expose the skull, and the skull surface was cleaned and moistened with sterile saline. Real-time CBF changes were recorded using a CCD camera. The same region of interest (ROI) (red circle) was defined for all groups. For the acute effect of FA, the CBF were measured for 10 min (baseline) and after the intervention (injection of FA) continuously for $50 \mathrm{~min}$. In the case of the measurements for the CBF in mice at 7 months old with the chronic FA or vehicle treatment, regional $\mathrm{CBF}$ was recorded throughout a 10-min period.

For measuring the blood flow of the jugular vein, C57 mice were under pentobarbital sodium (Sigma-Aldrich, $80 \mathrm{mg} / \mathrm{kg}$, i.p.) anesthesia, because the mice were fixed on the foam board with elastic that was not fit well with the respiratory anesthesia machine (isoflurane) and then given the neck skin discission to expose the jugular vein (slightly beat, $2-4 \mathrm{~mm}$ in diameter), and first added saline $(100 \mu \mathrm{L})$ directly to the exposed jugular vein and recorded for $5 \mathrm{~min}$ as the baseline, and then removed saline and added ET1 $(1 \mu \mathrm{g} / \mathrm{mL}, 100 \mu \mathrm{L})$ for $10 \mathrm{~min}$, and then removed the fluid and added saline $(100 \mu \mathrm{L})$ or FA $(2 \mathrm{mg} / \mathrm{mL}, 100 \mu \mathrm{L})$ for $10 \mathrm{~min}$. The droplets of saline, ET1, and FA were directly given to the exposed jugular vein through a pipette. Relative blood flow changes (\%) at the end of the observation period compared to the beginning of adding FA were calculated. 


\section{Ischemic Insult and 2,3,5-Triphenyltetrazolium Chloride Staining}

By using the techniques like those described previously $[50,51]$, the mice were injected with rose bengal $(100 \mathrm{mg} /$ $\mathrm{kg}$, i.p. Sigma-Aldrich) and $30 \mathrm{~min}$ later were anesthetized with sodium pentobarbital ( $80 \mathrm{mg} / \mathrm{kg}$, i.p, Sigma-Aldrich). The mice were under surgery with the body temperature $\left(37 \pm 0.5^{\circ} \mathrm{C}\right)$ maintained by a heating pad. The photo-thrombosis model of ischemic insult was induced in the hippocampus (AP, $-2.2 \mathrm{~mm}, \mathrm{ML} \pm 1.5 \mathrm{~mm}, \mathrm{DV}-1.3 \mathrm{~mm}$ ) by turning on the blue light for 20 -min (473 nm, Biogene, Beijing). Then, FA was given to the mice via intraperitoneal injection (i.p.) or intragastrical administration (i.g.). The 2,3,5-triphenyltetrazolium chloride (TTC) staining was used to measure the ischemic insult of the slices taken from the brain $24 \mathrm{~h}$ after the induction of the photo-thrombosis model in the hippocampus.

\section{The Time-of-Flight Magnetic Resonance Angiography}

The time-of-flight magnetic resonance angiography (MRA) was performed by using the small animal MRI 7.0 T (Bruker, Germany). Aesthesia of the mice was induced with a 1:4 mixture of oxygen and air using 2\% isoflurane (RWD Life Sciences, China). During the scan, each mouse was placed prone on a small animal MRI scanning bed. The head of the mouse was placed in the surface coil special for the head of the mouse and fixed by a tooth hook and double ear rods. The body coil and surface coil were used as the exciting and receiving coil. The inner diameter of the gantry is $16 \mathrm{~cm}$, and the head coil with an inner diameter of $38 \mathrm{~mm}$ was used. The anesthesia was maintained by the air mixed with $1.5 \%$ isoflurane through a nasal cannula of PE material during the scan. The temperature was warm and stable in the MRI scanning room. During the scanning process, the body temperature, respiratory rate, and heart rate of the mouse were monitored in real time by a physiological detector (SurgiVet V3395TPR, Smiths Medical, USA). The time-of-flight MRA scanning parameters are as follows: $\mathrm{TR}=12 \mathrm{~ms}, \mathrm{TE}=3 \mathrm{~ms}$, flip angle $=80^{\circ}, \mathrm{FoV}=20 \times 20 \mathrm{~mm}$, averages $=4$, slices $=80$, slice thickness $=0.2 \mathrm{~mm}$, time $=16 \mathrm{~min} 23 \mathrm{~s} 40 \mathrm{~ms}$.

The cerebral blood vessels were extracted by the iterative segmentation algorithm. In brief, (1) the initial threshold $\mathrm{T}_{0}$ was set as the mean value of the image; (2) the image was divided into the vessel and background parts according to the threshold; (3) the mean value of the two parts was calculated: mean ${ }_{\text {vessel }}$ and mean ${ }_{\text {background }}$; (4) the new threshold $T_{k}=\left(\operatorname{mean}_{\text {vessel }}+\right.$ mean $\left._{\text {background }}\right) / 2$. If $T_{0}=T_{k}$, the process is ended; otherwise, $T_{k}$ is set as the new threshold, and steps (2) to (4) were repeated. The newest $T_{k}$ was used to extract the vessel part $[52,53]$. After that, the vessel volume was obtained by the voxel numbers of the vessel part multiple by the volume of each voxel, and the blood vessel density was calculated by the vessel volume divided by the total intracranial volume.

\section{The Molecular Docking of Ferulic Acid on the Endothelin Receptor A}

The crystal structure of the human endothelin receptor typeB (ETRB) and ET-1 were obtained from RCSB Protein Data Bank (https://www.rcsb.org/) with PDB ID of 5GLH. The molecule SMILES of ferulic acid (FA) was obtained from the PubChem database (https://pubchem.ncbi.nlm.nih.gov/) with PubChem CID of 445858. Because there was no structure information of the endothelin receptor type A (ETRA) in PDB, SWISS-MODEL [54] was used to conduct protein structure homology-modeling of the ETRA. The input target sequence is the human ET1 receptor isoform a precursor (NCBI reference sequence: NP_001948.1) and ran with its default parameters. The SWISS-MODEL template library (SMTL version 2018-12-13, PDB release 2018-11-23) was searched with BLAST [55] and HHBlits [56] for evolutionary related structures matching the target sequence. The template with the highest quality (PDB ID: 5GLI) has then been selected for model building. Molecular docking analysis of FA with the ETRA was carried out with AutoDock Vina 1.1.2 [57]. All docking results were visualized and analyzed by using Discovery Studio 3.1.

\section{RNA Sequencing and Data Analysis}

The tissues from the hippocampus and the cortex of $\mathrm{AD}$ mice treated with drinking water containing FA (AD-FA) or $\mathrm{AD}$ and WT mice treated with the normal drinking water without FA were collected and stored in RNAlater (ThermoFisher) at $-80{ }^{\circ} \mathrm{C}$. Five mice in each group were used. RNA sequencing (RNA-seq) was performed by Novogene (Beijing, China). Briefly, the total RNA was extracted by using TRIzol reagent. Sequencing libraries were generated by using NEB. Next, Ultra RNA Library Prep Kit for Illumina (NEB, USA) following the manufacturer's recommendations and index codes were added to attribute sequences to each sample. Then, the products were purified (AMPure XP system) and library quality was assessed by the Agilent Bioanalyzer 2100 system. The clustering of the indexcoded samples was performed on a cBot Cluster Generation System using the HiSeq 4000 PE Cluster Kit (Illumia) according to the manufacturer's instructions. After cluster generation, the library preparations were sequenced on an Illumina Hiseq 4000 platform and 150 bp paired-end reads were generated.

The raw RNA-Seq data (paired-end reads) of the "fastq" format were processed with Skewer (version: 0.2.2) [58] 
for quality control and adapter trimming. Then, Pairfq was used (version: 0.17.0, https://github.com/sestaton/Pairfq) for reads pairing and removing the unpaired reads in each filtered paired-end file. The generated clean data with high quality were used for further analysis. Mouse reference genome and gene model annotation files (genome assembly: GRCm38.90) were downloaded from the Ensemble database (http://asia.ensembl.org/index.html). The genome index was built by using the python scripts included in the HISAT2 package (version: 2.1.0) [59, 60]. The pairedend clean reads were aligned to the reference genome by using HISAT2 and run with the default parameters. The aligned file in the "sam" format was sorted and converted to a "bam" format by using SAMtools (version 1.6) [61]. Transcripts assembly and estimating expression levels of the sorted reads were done by using StringTie (version 1.3.3b) [62] and run with the default settings. Next, the FPKM (fragments per kilobase of transcript per million fragments mapped) of each gene was calculated and summarized in the "Ballgown" package (version 2.2.0) [63].

The mouse gene annotation file was obtained by using the BioMart tool in the Ensemble database [64]. Gene annotation of the processed RNA-Seq file was made by using custom-written $\mathrm{R}$ scripts. There were 44 types of RNAs in the annotated expression matrix for which mRNAs were extracted (the type of "protein coding") for further analysis. Since the data contain two brain regions from three groups, we retained a gene with a $\log 2$ FPKM expression greater than 0.1 in more than 3 samples per group to ensure the data quality. Finally, we got an expression matrix with 12,466 unique genes and 36 samples.

The R statistical software v3.4.1 was used to perform data analysis. Differentially expressed gene analysis was performed by using the empirical Bayes algorithm in the "limma" package [65] in R. Differences (up- or downregulated) were considered statistically significant if the absolute value fold changes higher than 1.5 and the $P$ values $\leq 0.05$. Differentially expressed genes were calculated between $\mathrm{AD}$ vs. WT; AD-FA vs. AD; AD-FA vs. WT in both the hippocampus and the cortex. The expression profiles of the union set of differentially expressed genes in the three comparisons were showed by heatmap in the "pheatmap" package. We chose the "ward.D2" algorithm to perform hierarchical clustering for samples in different groups.

The gene sets of GO biological processes were downloaded from the QuickGO database (https://www.ebi.ac. uk/QuickGO/). We filtered the gene sets correlated to specific functions including amyloid, astrocyte, microglia, synapse, vascular, and immune-related functions, or signal pathways. We mainly focused on the expression changes in the above gene sets between AD and WT, and AD and AD-FA.

\section{Statistical Analysis}

All data are represented as mean \pm SEM. All analyses were performed using GraphPad Prism 7. Data were analyzed by using unpaired Student's $t$-test for two independent groups, paired one for before and after comparison, and one-way or two-way ANOVA among groups followed by multiple comparisons. The significance level was set at $P<0.05$.

\section{Results}

\section{Reduction of Diameter and Density of Hippocampal Capillary Precedes A $\beta$ Plaque Deposition and Spatial Memory Decline in APP/PS1 Mice}

We first assessed AD and WT mice using the spatial learning task of the Morris water maze [35], a type of episodic memories. We found that $\mathrm{AD}$ mice displayed a progressive worse decline of memory than WT mice did, as manifested by spatial learning over 5-day training and spatial memory tested $24 \mathrm{~h}$ after the learning, both of which were unaffected from 3 to 4 months old, but mildly impaired from 6 to 7 months old, and more impaired from 9 to 11 months old, relative to WT mice (Fig. 1a, b, Spatial learning; 3 to 4 months old: $N=14$ / group, $F_{(1,26)}=1.074, P=0.309 ; 6$ to 7 months old: $N=12 /$ group, $F_{(1,22)}=7.539, * P=0.011 ; 9$ to 11 months old: $N=11$ for WT, $N=12$ for AD, $F_{(1,21)}=5.838, * P=0.024$; repeated measure ANOVA. Spatial memory. Group, $F_{(1,69)}=13.680, P<0.001$; age, $F_{(2,69)}=2.184, P=0.120$; group $\times$ age, $F_{(2,69)}=0.803, P=0.452$. Holm-Sidak's analysis for WT vs. AD: 3 to 4 months old, $P=0.229 ; 6$ to 7 months old, $* P=0.025 ; 9$ to 11 months old, $* P=0.035$; two-way ANOVA).

Immunostaining for $A \beta$ plaque was then performed. $\mathrm{AD}$ mice showed a progressive load of $\mathrm{A} \beta$ plaques in the hippocampus, as demonstrated no $A \beta$ plaques at 4 months old, but scattered $A \beta$ plaques at 7 months old, and more $\mathrm{A} \beta$ plaques at 9 months old, relative to WT mice (Fig. 1c, d, 4 months old: WT, $n=8(N=3)$; AD, $n=9(N=3)$; 7 months old: WT or AD, $n=10(N=3)$; 9 months old: WT or AD, $n=12(N=3)$; group, $F_{(1,55)}=110.9, P<0.001$; age, $F_{(2,55)}=55.73, P<0.001$; group $\times$ age, $F(2,55)=53.74$, $P<0.001$; Holm-Sidak's analysis for 4 months old: WT $v$ s. $\mathrm{AD}, P=0.910$; for 7 months old: WT vs. $\mathrm{AD}, * * * P<0.001$; for 9 months old: WT $v s$. AD, $* * * P<0.001$; two-way ANOVA).

We then used collagen IV immunostaining to measure capillary changes in the hippocampus where $\mathrm{A} \beta$ plaques were first found. Notably, the capillaries in AD mice exhibited a progressive worse reduction than those in WT mice did, as indicated by unaltered density but reduced diameter since 4 months old, but reduction of density and diameter 
Fig. 1 Reduced hippocampal capillaries precede $\mathrm{A} \beta$ plaque deposition and spatial memory deficit in APP/PS1 mice. (a) Spatial learning indicated by latency (s) onto a hidden platform in WT (black circle) vs. AD mice (blue circle) was not different until 6 to 7 months old. (b) Spatial memory was not different until 6 to 7 months old. (c) Representative images for hippocampal $\mathrm{A} \beta$ plaques using the antibody $6 E 10$ in $\mathrm{AD}$ mice. (d) $\mathrm{A} \beta$ plaque area (\%) indicated a sharp increase of $A \beta$ plaque load since 7 months old in $\mathrm{AD}$ mice. (e) Representative images for hippocampal capillaries using the antibody $\mathrm{Col}$ lagen IV in $\mathrm{AD}$ and $\mathrm{WT}$ mice. $(\mathbf{f}, \mathbf{g})$ Capillary density (\%) and diameter $(\mu \mathrm{m})$ suggested an age-dependent reduction of hippocampal capillaries in $\mathrm{AD}$ mice relative to WT mice. Data are presented as mean \pm SEM. $* P<0.05, * * P<0.01$, $* * * P<0.001$ a
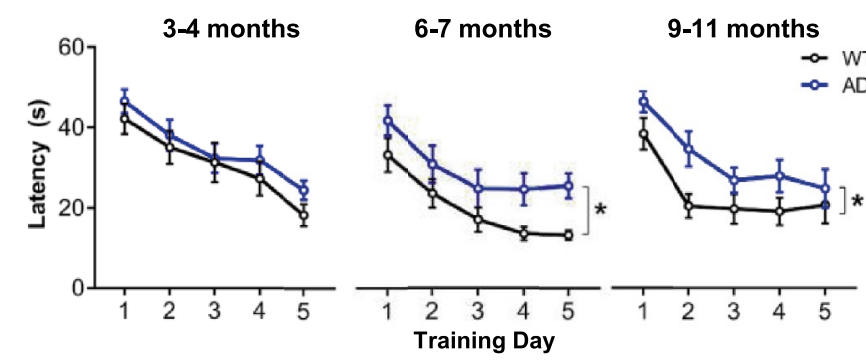

C
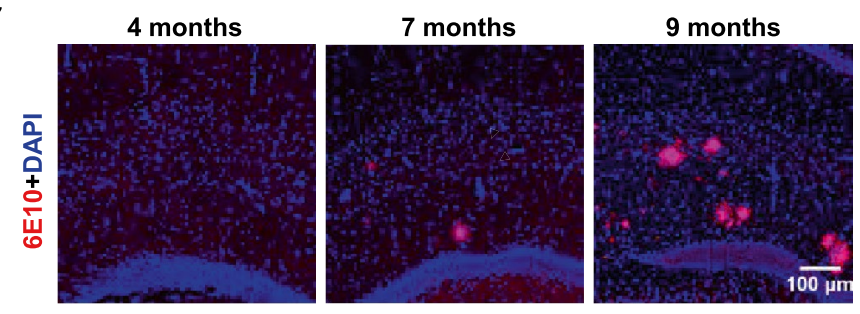

e

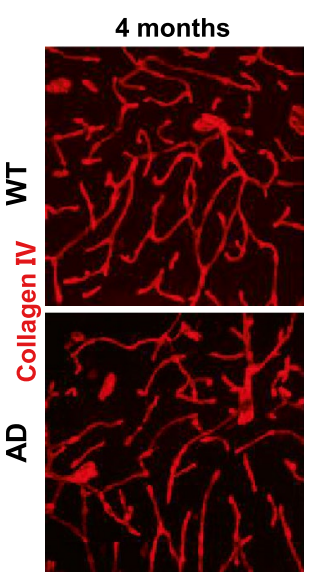

b

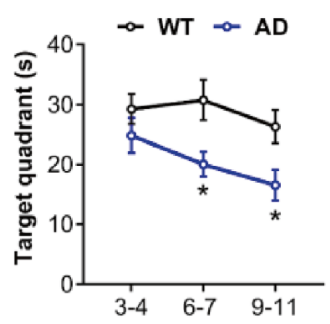

d
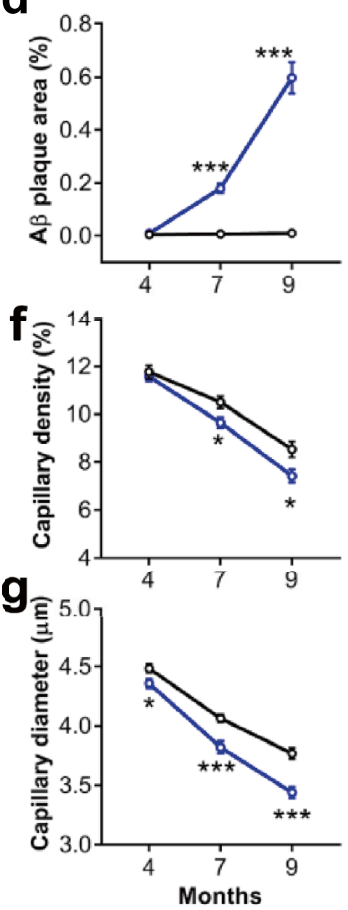

at 7 months old and more reduction of the both at 9 months old, relative to WT mice (Fig. 1e-g, Capillary density: 4 months old: WT or AD, $n=18(N=3)$; 7 months old: WT or $\mathrm{AD}, n=18(N=3)$; 9 months old: WT, $n=17(N=3)$; $\mathrm{AD}, n=16(N=3)$; group, $F_{(1,99)}=11.61, P=0.001$; age, $F_{(2,99)}=101.5, P<0.001 ;$ group $\times$ age, $F_{(2,99)}=1.664$, $P=0.194 ;$ Holm-Sidak's analysis for 4 months old: WT $v s$. $\mathrm{AD}, P=0.589$; for 7 months old: WT vs. AD, $* P=0.039$; for 9 months old: WT vs. AD, $* P=0.011$. Capillary diameter: $n=18(N=3)$ /group for each age; group, $F_{(1,102)}=45.40, P<0.001$; age, $F_{(2,102)}=187.2, P<0.001$; group $\times$ age, $F_{(2,102)}=2.838, P=0.0632 ;$ Holm-Sidak's analysis for 4 months old: WT vs. AD, $* P=0.035$; for 7 months old: WT $v s$. AD, $* * * P<0.001$; for 9 months old: WT vs. AD, $* * * P<0.001$; two-way ANOVA). This result suggests that that the earliest change is the reduced diameter of capillaries at 4 months old, and the later change is the reduced diameter and density of capillaries at 7 months old when $A \beta$ plaque deposition and spatial memory deficit are observed.
These findings are particularly interesting because we found that changes of hippocampal capillaries could be long before the onset of $A \beta$ plaque deposition and memory decline [66], probably consistent with the neurovascular hypotheses of $\mathrm{AD}[19,67]$.

The ultrastructures associated with hippocampal capillaries from AD and WT mice aged 3 to 12 months old were also examined using transmission electron microscopy (Supplementary Fig. 1a). The extra-capillary astrocytic end feet might develop edema, and the glial pedal plate could begin mild degeneration in AD mice aged 6 months old (Supplementary Fig. 1b). Other ultrastructures such as the thickness of the perivascular basement membrane, the tight junction between the endothelial cells, and the pericyte on the outside of the capillaries were detected not a difference between $\mathrm{AD}$ and WT mice from 3 to 6 months old (Supplementary Fig. 1c-e).

Hippocampal microglia and astrocyte were further examined. Immunostaining of microglia showed that the total number of microglial cells was not different between AD 
and WT mice at 4 months old but increased in AD mice at 7 months old or 9 months old (Supplementary Fig. 2a, b). However, activated microglial cells were distributed in proximity to $A \beta$ plaques and may release proinflammatory factors. Thus, three or more microglial cells clustered together were defined as "aggregative microglial cells" (Supplementary Fig. 2a, yellow box). We found that the area of aggregative microglial cells was increased in AD mice since the 7 months old (Supplementary Fig. 2c). The astrocytic area showed no difference between AD and WT mice at 4 or 7 months old; it was increased in AD mice at 9 months old (Supplementary Fig. 2d, e). Furthermore, the increased number of microglial cells at 7 months old was confirmed by using DAB staining (Supplementary Fig. 2f, g).

Together, AD mice showed the early changes of the hippocampus from 4 to 7 months old: the earliest one was the reduced diameter of hippocampal capillaries at 4 months old, and the later ones were the reduced density and diameter of hippocampal capillaries, hippocampal A $\beta$ plaque deposition, the increased number of microglial cells and increased area of aggregative microglial cells, and spatial memory deficit at 7 months old. Other changes were either not significant or later than this age.

\section{ET1 Induces Early A $\beta$ Plaque Deposition}

The reduced density and diameter of hippocampal capillaries are age-dependent and could have caused inconspicuous tiny hypoperfusion. This hypoperfusion can be mimicked by the injection of ET1 into the hippocampus [68]. Therefore, to address whether reduced density/diameter of hippocampal capillaries could be sufficient for initiating $\mathrm{A} \beta$ plaque deposition, we injected ET1 or vehicle into hippocampal CA1 region in WT or AD mice aged 5 months old (Fig. 2a) when there are no $A \beta$ plaques at this young age. Lectin perfusion and imaging revealed a small hypoperfusion insult in WT or AD mice 7 days after the ET1 injection (Fig. 2b). Notably, the staining of $\mathrm{A} \beta$ plaques using either the antibody $D 54 D 2$ or Thioflavine $S$ similarly revealed that ET1 caused early A $\beta$ plaque deposition in the ET1 but not the vehicle group in $\mathrm{AD}$ mice. In contrast, $\mathrm{A} \beta$ plaques were nearly undetectable in both the ET1 and control groups in WT mice (Fig. 2c-e, $n$ $=9(N=3) /$ group; $A \beta^{+}$area for Vehicle $v s$. ET1: AD mice, $* * * P<0.001$; WT mice, $P=0.347$; Ths $^{+}$area for Vehicle vs. ET1: AD mice, ${ }^{* * *} P<0.001$; WT mice, $P=0.766$; twoway ANOVA).

These results suggest that reduced density and diameter of hippocampal capillaries mimicked by the injection of ET1 into the hippocampus could be sufficient for initiating $A \beta$ plaque deposition, consistent with the neurovascular hypotheses $[19,67]$, for which vascular damage can increase $A \beta$ accumulation [29-33] or decrease $A \beta$ clearance [67].

\section{FA Prevents Reduction of Both CBF and Density/ Diameter of Hippocampal Capillaries in APP/PS1 Mice}

To address whether reduced density and diameter of hippocampal capillaries could be also necessary for initiating $\mathrm{A} \beta$ plaque deposition, we used FA (or 4-hydroxy-3-methoxycinnamic acid) (Fig. 3a), because previous studies have suggested its antioxidant or anti-inflammatory actions to be potential for treating $\mathrm{AD}[37,38]$ and because we found that intraperitoneal injection (i.p.) or intragastric administration (i.g.) of FA alleviated hypoperfusion insult in the hippocampus with a dose-dependent effect (Supplementary Fig. 3). Notably, we found that FA could antagonize the ET1-mediated vasoconstriction, as examined using laser speckle imaging on the blood flow: applying ET1 evoked strong vasoconstriction; following application of FA induced vasodilation (Fig. 3b, c, ETI-Saline, $N=5$; ET1-FA, $N=4$; $F_{(1,63)}=31.15, * * * P<0.001$; two-way ANOVA). Compared with saline, following application of FA counteracted the ET1-mediated vasoconstriction (Fig. 3d, Saline, $N=5$; FA, $N=4 ; t=4.165, d f=7$, **P=0.004; Student's $t$-test). Furthermore, molecular docking analysis revealed that FA could bind on the ETRA, the pharmacological target of ET1, with a high affinity (Supplementary Fig. 4a, b). Besides, the synthesized FA-biotin was co-localized mainly with the ETRA on the blood vessels of the hippocampus (Supplementary Fig. 4c).

Therefore, we further investigated if FA could increase $\mathrm{CBF}$ in $\mathrm{AD}$ mice. A single treatment of FA (i.p., $10 \mathrm{mg} /$ $\mathrm{kg}$ ) induced a significant increase of CBF in C57 or AD mice (Fig. $3 e$, f, $N=4$ /group; $C 57$ mice: dose, $\left.F_{(1,6)}\right)=9.988$, $* P=0.019$; Time, $F_{(6,36)}=4.603, P=0.001$; dose $\times$ Time, $F_{(6,36)}=2.348, P=0.051$. AD mice: dose, $F_{(1,6)}=15.69$, $* * P=0.007$; Time, $F_{(6,36)}=1.548, P=0.190$; dose $\times$ Time, $F_{(6,36)}=1.031, P=0.421$. Two-way ANOVA). Thereafter, we administered FA to AD mice chronically, started at 6 months old via daily drinking water for 30 days (averaged about $20 \mathrm{mg} / \mathrm{kg} /$ day, termed as AD-FA), while the normal drinking water without FA as the vehicle controls (AD or WT mice). $\mathrm{CBF}$ in $\mathrm{AD}$ mice with the vehicle treatment (AD, Fig. 3g) was lower than that in WT mice aged 7 months old, but this $\mathrm{CBF}$ reduction was prevented by the FA treatment (AD-FA, Fig. 3h, WT, $N=13$; AD or AD-FA, $N=11$; $F_{(2,32)}=5.773, P=0.007$; WT $v s . \mathrm{AD}, * * P=0.006$; WT vs. AD-FA: $P=0.738$; AD vs. AD-FA: $P=0.051$. One-way ANOVA followed by Tukey's analysis). Notably, immunostaining of capillaries (Fig. 4a) showed that this 30-day FA treatment increased capillary density (Fig. 4b, hippocampus: $\mathrm{AD}, n=13(N=4)$; AD-FA, $n=16(N=5) ; t=3.13$, $d f=27, * * P=0.004$; cortex: AD, $n=13(N=4)$; AD-FA, $n=15(N=5) ; t=2.65, d f=26,{ }^{*} P=0.013$; Student's $t$-test $)$ and diameter (Fig. 4c, hippocampus: $\mathrm{AD}, n=12(N=4)$; 
Fig. 2 ET1 induces a tiny hypoperfusion and early $\mathrm{A} \beta$ plaque deposition at 5 months old. (a) Procedures: ET1 or vehicle injection $(1 \mu \mathrm{L}, 0.1 \mu \mathrm{L} /$ min, $1 \mu \mathrm{g} / \mu \mathrm{L})$ into one side of hippocampal CA1. (b) Lectin perfusion and imaging revealed a hypoperfusion insult in the ET1 group but not the vehicle group. (c) Representative images for $\mathrm{A} \beta$ plaque using the antibody $D 54 D 2(\mathrm{~A} \beta)$ or Thioflavine $S$ (Ths) in AD mice. (d, e) $\mathrm{A} \beta^{+}$or $\mathrm{Ths}^{+}$area $\left(\mu \mathrm{m}^{2}\right)$ indicated early $\mathrm{A} \beta$ plaque deposition in the ET1 group but not the vehicle group in $\mathrm{AD}$ mice, nearly undetectable in both the groups in WT mice. Data are presented as mean \pm SEM. $* * * P<0.001$ a

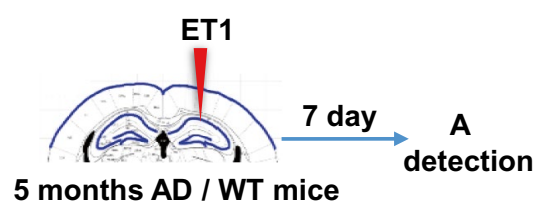

C
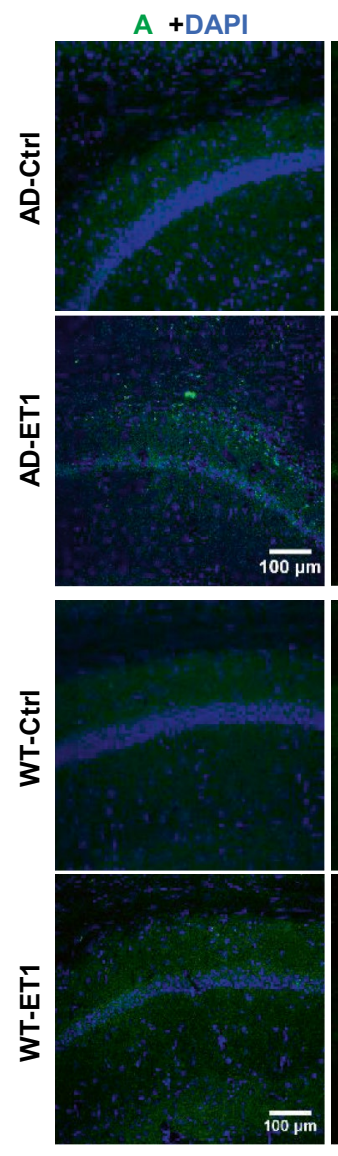

d

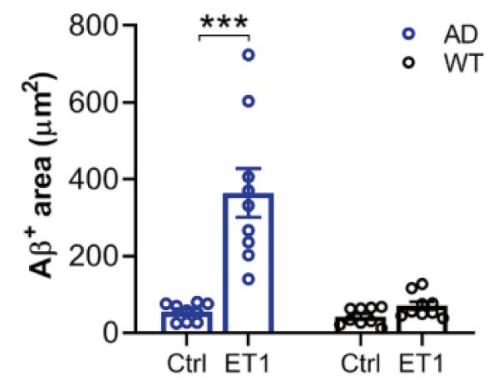

b

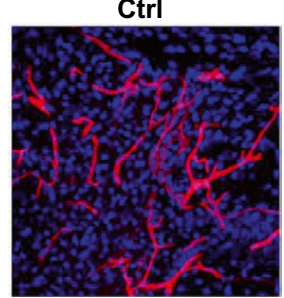

A
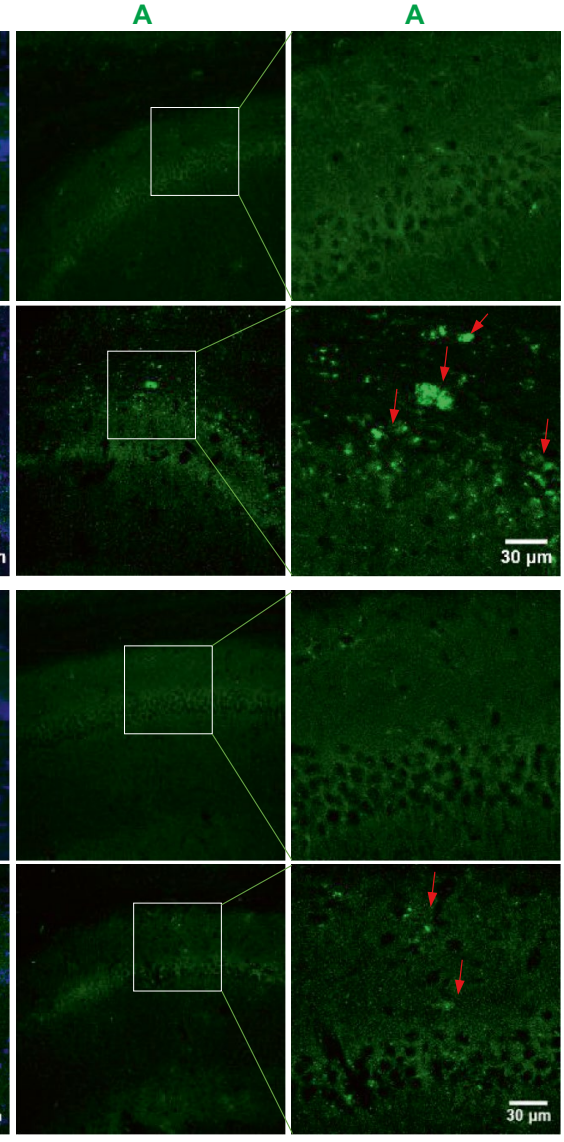

e

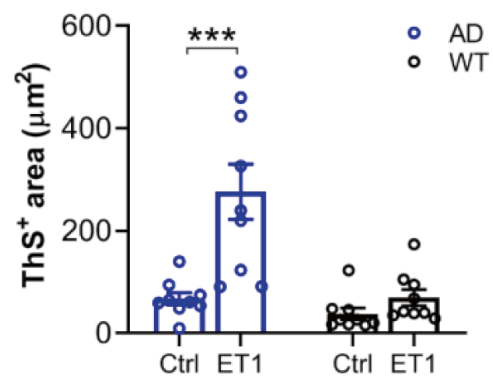

AD-FA, $n=15(N=5) ; t=4.951, d f=25$, *** $P<0.001$; cor tex: $\mathrm{AD}, n=12(N=4) ; \mathrm{AD}-\mathrm{FA}, n=15(N=5) ; t=2.778$, $d f=25, * P=0.010$; Student's $t$-test $)$ in the hippocampus and cortex.

Furthermore, a non-intrusive method, i.e., the time-offlight magnetic resonance angiography (MRA), was used to measure the potential changes of the whole-brain blood vessel density. AD mice were subjected to a 7-T MRA scan at 6 months old and underwent a similar paradigm of the FA or vehicle treatment for 30 days, and subjected to the 7-T MRA scan again at 7 months old. We found that the density of the whole-brain blood vessels was increased in the AD-FA group as compared with the pre-treatment baseline, but not in the vehicle-treated AD group (Fig. 4d, e, 
a<smiles>COc1cc(/C=C/C(=O)O)ccc1O</smiles>

Ferulic acid b

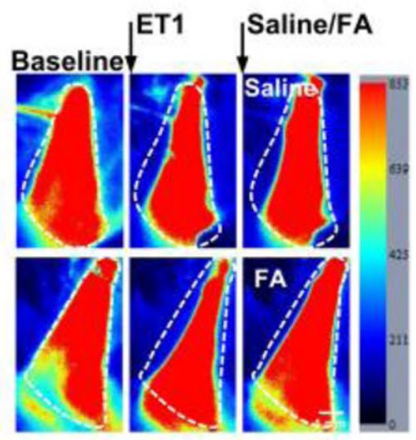

C

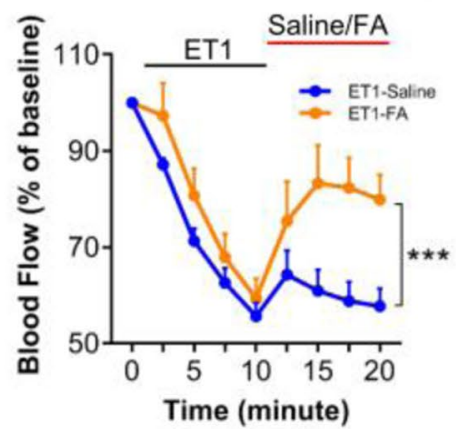

d

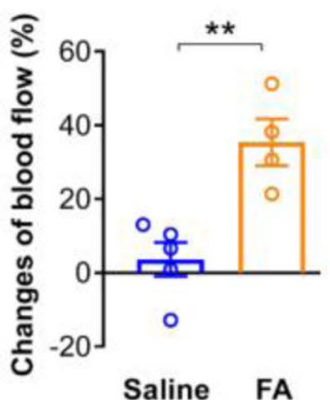

e

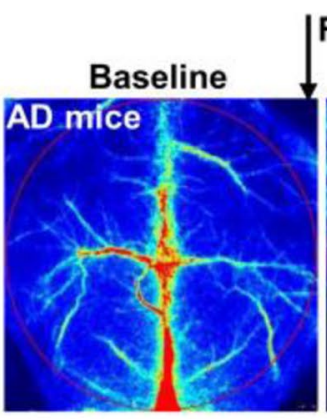

$\mathrm{FA}(10 \mathrm{mg} / \mathrm{kg}$, i.p)

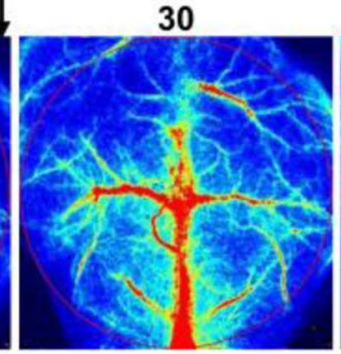

g
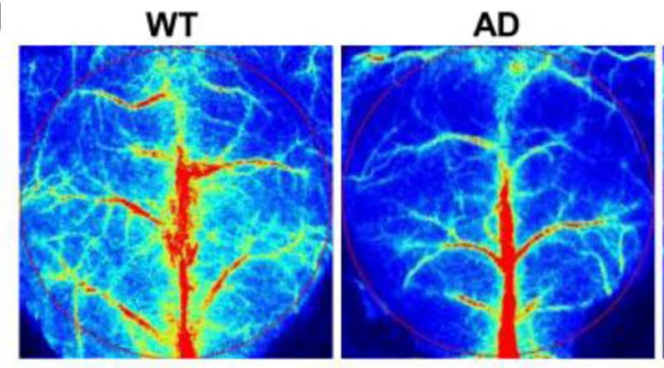

AD-FA

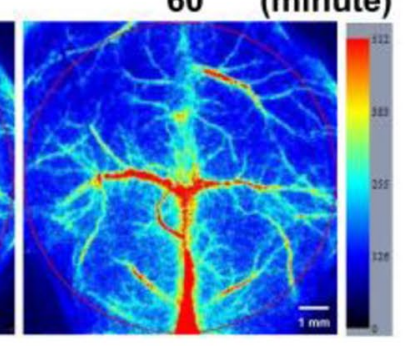

f

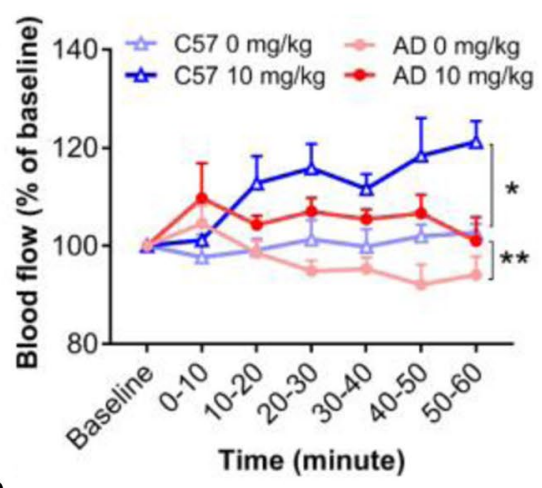

h
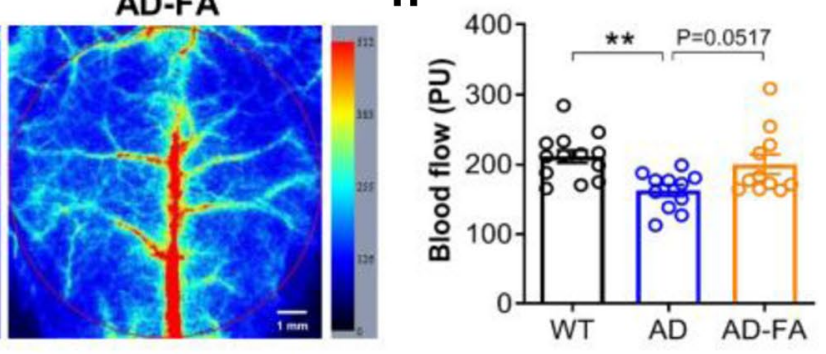

Fig. 3 FA prevents the reduction of the CBF. (a) Chemical structure of ferulic acid (FA). (b) Experimental procedures and representative images of laser speckle imaging for the blood flows in the jugular vein. (c) ET1 $(1 \mu \mathrm{g} / \mathrm{mL}, 100 \mu \mathrm{L})$ induced strong vasoconstriction as indicated by the reduction of the blood flows, while following application of FA $(2 \mathrm{mg} / \mathrm{mL}, 100 \mu \mathrm{L})$ but not vehicle (Saline) induced vasodilation in C57 mice at 2 months old. (d) Relative changes (\%) of the blood flows before and after adding FA or saline for $10 \mathrm{~min}$ indicated that FA counteracted the ET1-mediated action. (e) Representative images of laser speckle imaging in $\mathrm{AD}$ mice at 6 months old after intraperitoneal injection (i.p.) of FA $(10 \mathrm{mg} / \mathrm{kg})$. (f) The CBF was increased by FA $(10 \mathrm{mg} / \mathrm{kg}$, i.p.) in AD or C57 mice. (g, h) The FA treatment rescued the $\mathrm{CBF}$ reduction in $\mathrm{AD}$ mice that was observed in the vehicle group, relative to WT mice, by using laser speckle imaging at 7 months old. The same region of interest (ROI) (red circle) was defined for all groups
AD, $N=10$; AD-FA, $N=9 ; A D: t=0.208, d f=9, P=0.839$; $A D$-FA: $t=2.355, d f=8, * P=0.046$. Paired $t$-test). This finding strongly suggests that FA treatment is beneficial for increasing the density of the whole-brain blood vessels in AD mice, highly consistent with the above studies for density and diameter of capillaries.

\section{FA Prevents A $\beta$ Plaque Deposition Partially}

We then tested if FA could prevent $\mathrm{A} \beta$ plaque deposition in $\mathrm{AD}$ mice. $\mathrm{A} \beta$ plaque deposition in the hippocampus and cortex in AD mice at 7 months old was also largely prevented by the FA treatment relative to the vehicle controls (Fig. 5a, b, hippocampus: AD, $n=29(N=5)$; AD-FA, $n=30(N=5) ; t=2.604, d f=57,{ }^{*} P=0.011$; Cortex: AD, $n=26(N=5)$; AD-FA, $n=25(N=5) ; t=3.131, d f=49$, $* * P=0.002$. Student's $t$-test). These effects of FA were likely achieved by reducing the accumulation of $A \beta$ in the hippocampus because the ELISA test showed a significant reduction of the $A \beta 1-42$ and a downtrend of the $A \beta 1-40$ in the hippocampus from $\mathrm{AD}$ mice with the FA treatment relative to the vehicle controls (Fig. 5c, d, AD, N=4; AD-FA, 
Fig. 4 FA prevents the reduction of hippocampal capillaries in $\mathrm{AD}$ mice at 7 months old. (a) Representative images for immunostaining of the blood vessel (Collagen IV) in the hippocampus and cortex in $\mathrm{AD}$ mice after the FA or vehicle treatment. (b, c) Quantification of capillary density (\%) (b) and diameter $(\mu \mathrm{m})(\mathbf{c})$ in the hippocampus and the cortex suggested that the both were significantly increased by the FA treatment relative to the vehicle control. (d) Representative 2D time-of-flight images for blood vessels in $\mathrm{AD}$ mice before and after the FA treatment or vehicle control. (e) Comparison of the time-of-flight magnetic resonance angiography between before and after the treatment suggested that the FA treatment but not the vehicle control significantly increased the density of the whole-brain blood vessels. Data are presented as mean \pm SEM. $* P<0.05$, $* * P<0.01$ a

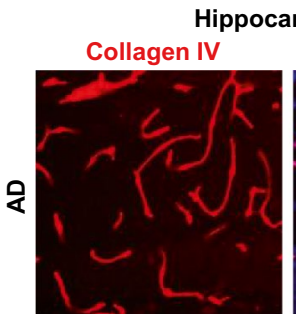
Collagen IV+DAPI
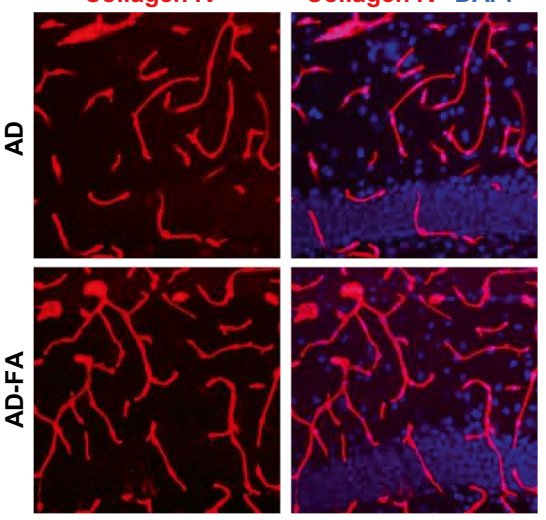

b
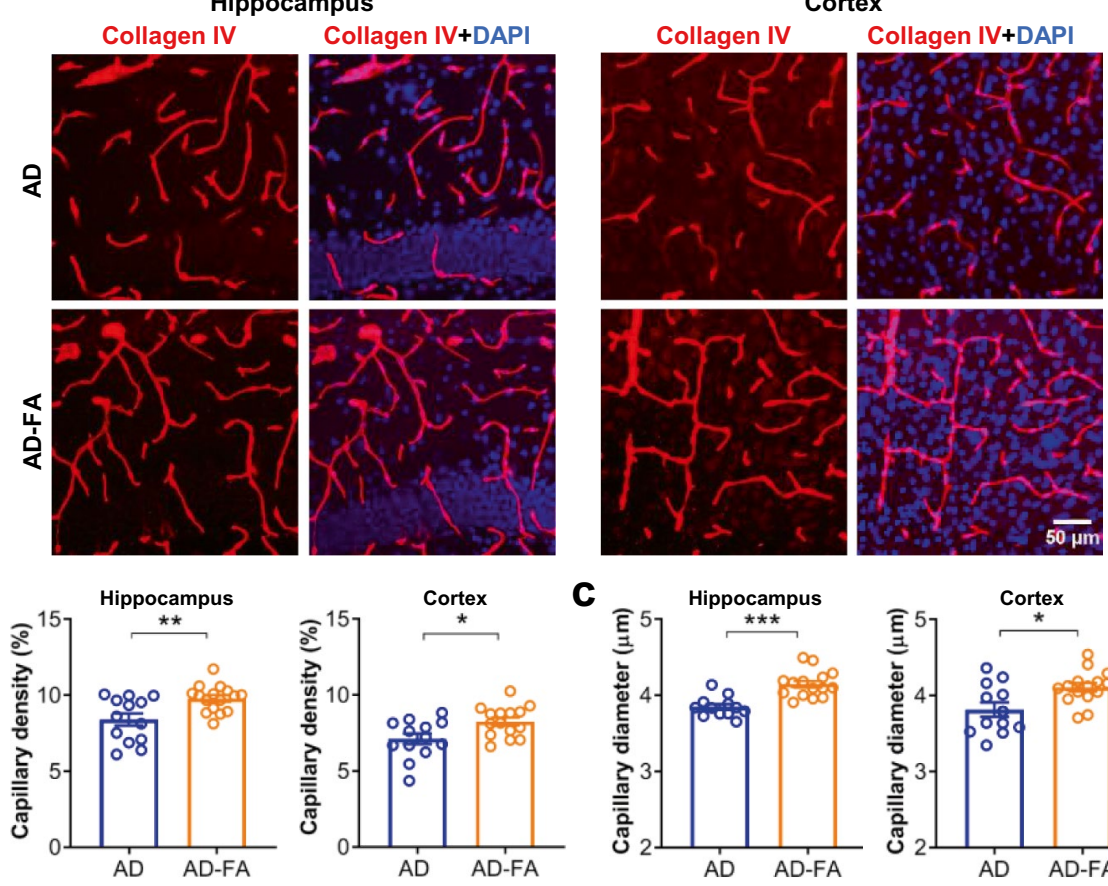

d
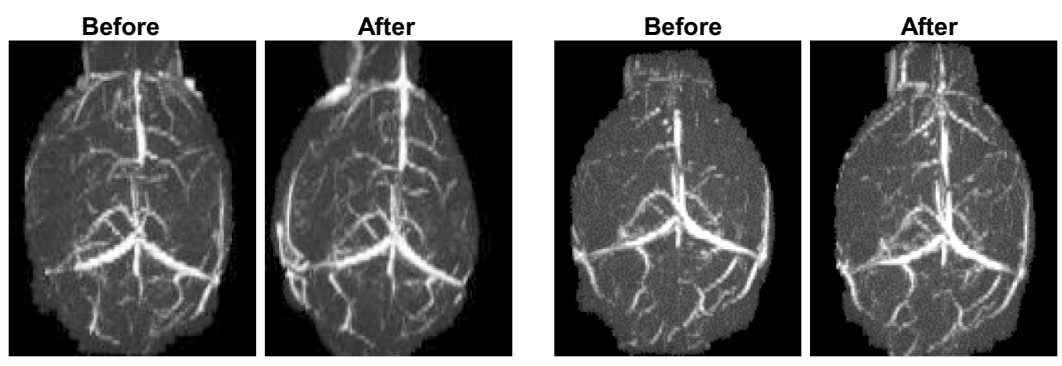

$\mathrm{AD}$

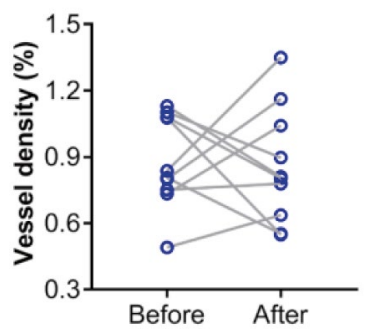

AD-FA

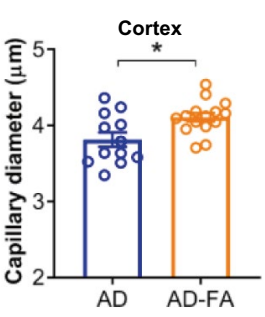

After

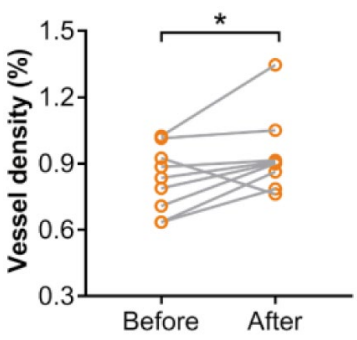

$N=5 ; A \beta 42: t=3.145, d f=7,{ }^{*} P=0.016 ; A \beta 40: t=2.136$, $d f=7, P=0.070$. Student's $t$-test). Likewise, the early A $\beta$ plaque deposition in the hippocampus, initiated 7 days after the hippocampal injection of ET1 at 5 months old, was persistent up to 6 months old with the vehicle treatment, but that was partially prevented by the FA treatment, reduced to a level about $50 \%$ of the vehicle controls (Fig. $5 \mathrm{e}, \mathrm{f}, \mathrm{AD}$, $n=20(N=7)$; AD-FA, $n=22(N=7) ; t=2.987, d f=40$, $* * P=0.005$. Student's $t$-test). These reduced A $\beta$ levels in the hippocampus were not attributable to a change of APP expression because western blot of the hippocampal tissues from AD mice showed no significant changes in the total levels of APP protein between the FA and vehicle groups
(Supplementary Fig. 5a). Also, the $\beta$-site of the APP cleaving enzyme 1 (BACE1) activity, measured by using the $\beta$-secretase activity fluorometric assay kit, was increased in the hippocampus from AD mice with the vehicle treatment but was backed to the WT levels by the FA treatment (Supplementary Fig. 5b). Consistent with earlier reports, FA or FA-based hybrid treatment reduced BACE1 expression and $\beta$-secretase activity in AD mice or cultured APP-overexpressing murine neuron-like cells [69]. The possible mechanism is that FA rescued the hypoxia-up-regulated BACE1 activity [29] and/or inhibited the NFKB-dependent transcription of BACE1 [70]. It has been also reported that FA may directly interact with BACE1 and inhibit its activity [71]. 
Fig. 5 FA reduced $\mathrm{A} \beta$ plaque deposition in $\mathrm{AD}$ mice at 7 months old and ET1-induced early $\mathrm{A} \beta$ deposition. (a) Representative images for immunostaining of the $A \beta$ antibody (6E10) in $\mathrm{AD}$ mice at 7 months old after the FA (AD-FA) or vehicle treatment (AD) for 30 days. (b) Quantification of the $A \beta$ area $\left(\mu \mathrm{m}^{2}\right)$ in the hippocampus and the cortex suggested a significant reduction of $A \beta$ plaques by the FA treatment relative to the vehicle control. (c, d) The enzyme-linked immunosorbent assay test suggested a significant reduction of the $A \beta 1-42$ (e) and a downtrend of the $\mathrm{A} \beta$ $1-40$ (f) concentration from AD mice after the FA treatment relative to the vehicle control. (e) Representative images for immunostaining of the early A $\beta$ plaques $(D 54 D 2)$ initiated by the injection of ET1 into the hippocampal CA1 region in $\mathrm{AD}$ mice at 5 months old and underwent the ferulic acid (FA) treatment (AD-FA) or vehicle treatment (AD) for 30 days, started from the same day with the ET1 injection. (f) Quantification of the $A \beta$ area $\left(\mu m^{2}\right)$ suggested that the FA treatment significantly reduced the ET1-induced early A $\beta$ plaque deposition relative to the vehicle treatment a

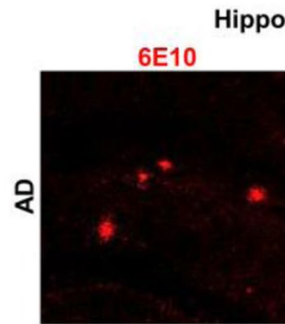

Hippocampus

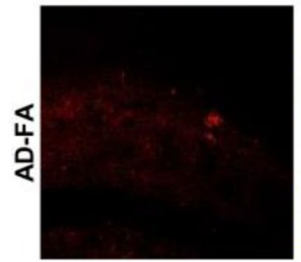

b
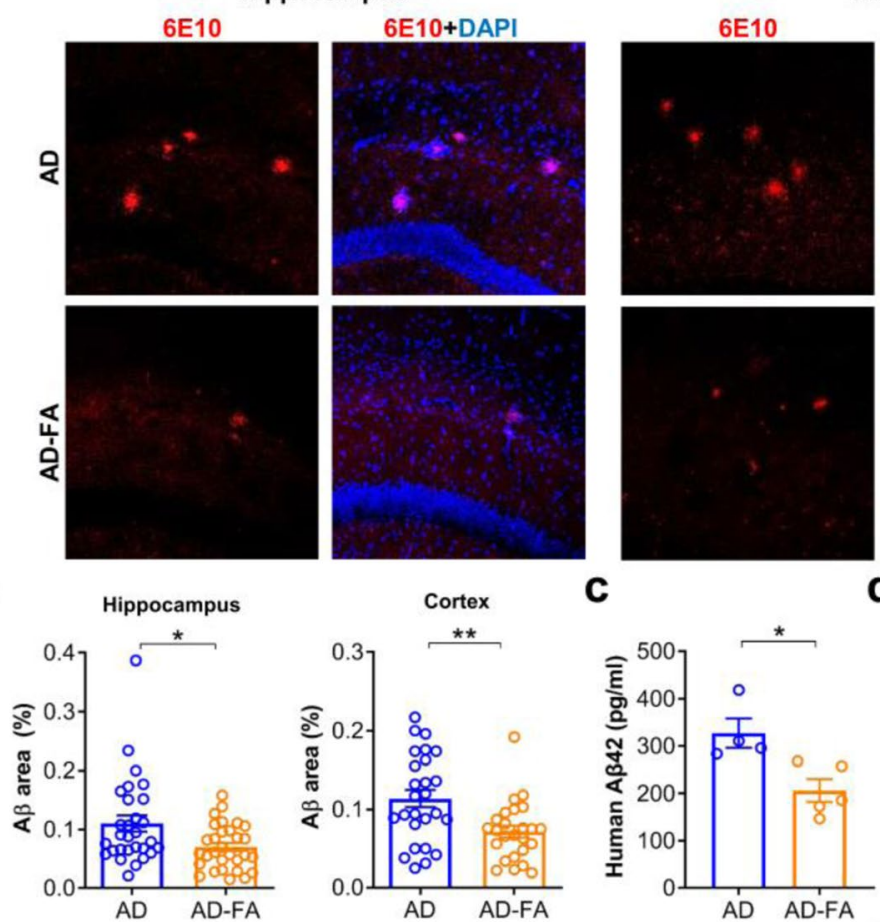

Cortex

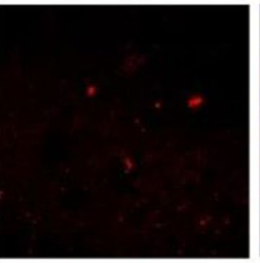

C
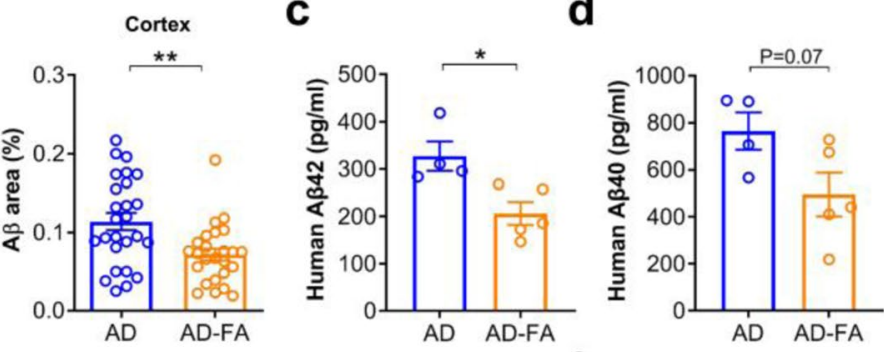

e
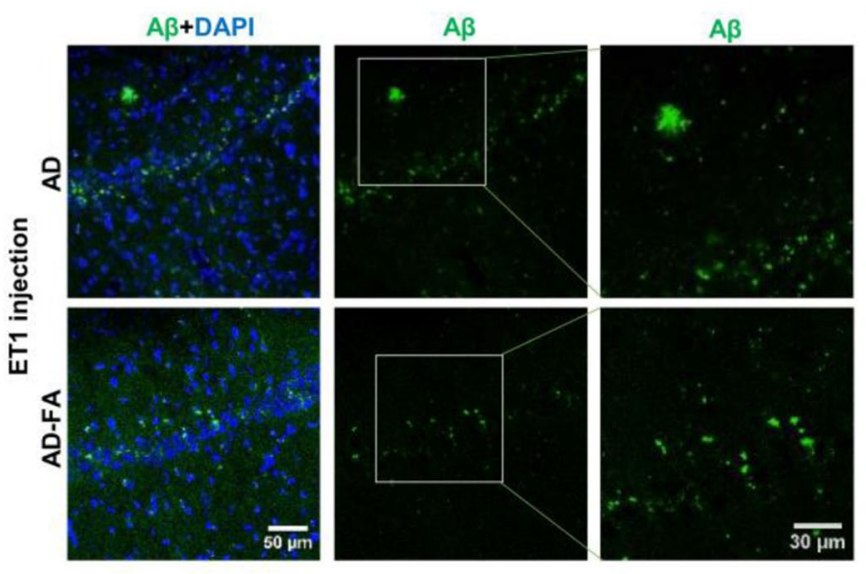

f
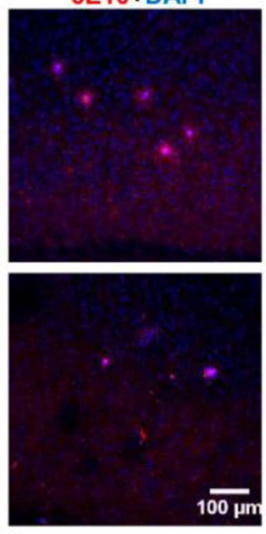

d
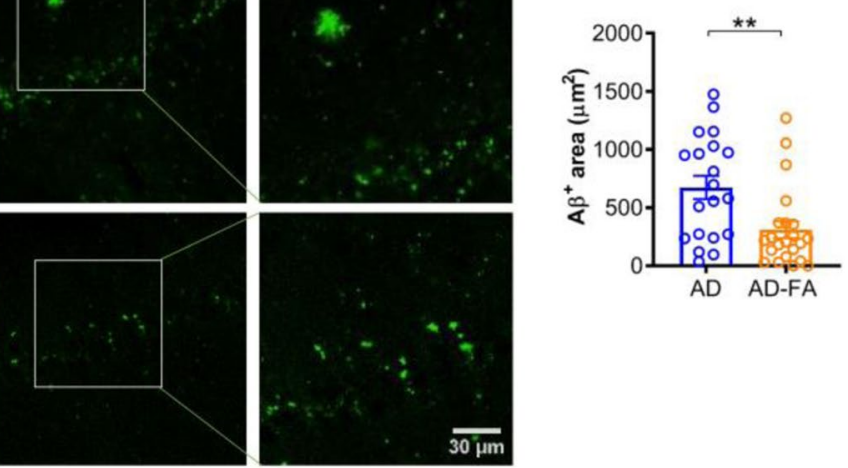

We next performed co-immunostaining of $\mathrm{A} \beta$ plaque deposition with microglia in the hippocampus from $\mathrm{AD}$ mice at 7 months old after the 30-day FA or vehicle treatment. No significant differences were found in the total number of microglial cells, but the area of the aggregative microglial cells, characterized by more than 3 cells aggregated proximity to the $A \beta$ plaques, was significantly reduced after the FA treatment relative to vehicle control (Supplementary Fig. 6a-c). In contrast, immunostaining for astrocyte showed that the FA treatment produced no significant effect on the astrocytic area (Supplementary Fig. 6d-f), due to no changes at this age (see 7 months old in Supplementary Fig. 2d, e).

\section{FA Prevents Spatial Memory Deficit Completely}

Finally, we examined if FA could prevent spatial memory deficit in AD mice from 6 to 7 months old. AD mice with the 30-day FA treatment performed the spatial learning task significantly better than those with the vehicle treatment did, as reflected by a shorter latency in escaping onto a hidden platform during 5-day training (Fig. 6a, WT, $N=12$; AD or AD-FA, $N=13$; group, $F_{(2,35)}=4.429, P=0.019$; day, $F_{(4,140)}=32.82, P<0.001$; group $\times$ day, $F_{(8,140)}=1.64$, $P=0.118$; post hoc: WT $v s$. AD, $* P=0.034$; WT $v s$. AD-FA: $P=0.988$; AD $v s$. AD-FA: $* P=0.042$. Two-way ANOVA followed by Tukey's post hoc), suggesting the full 


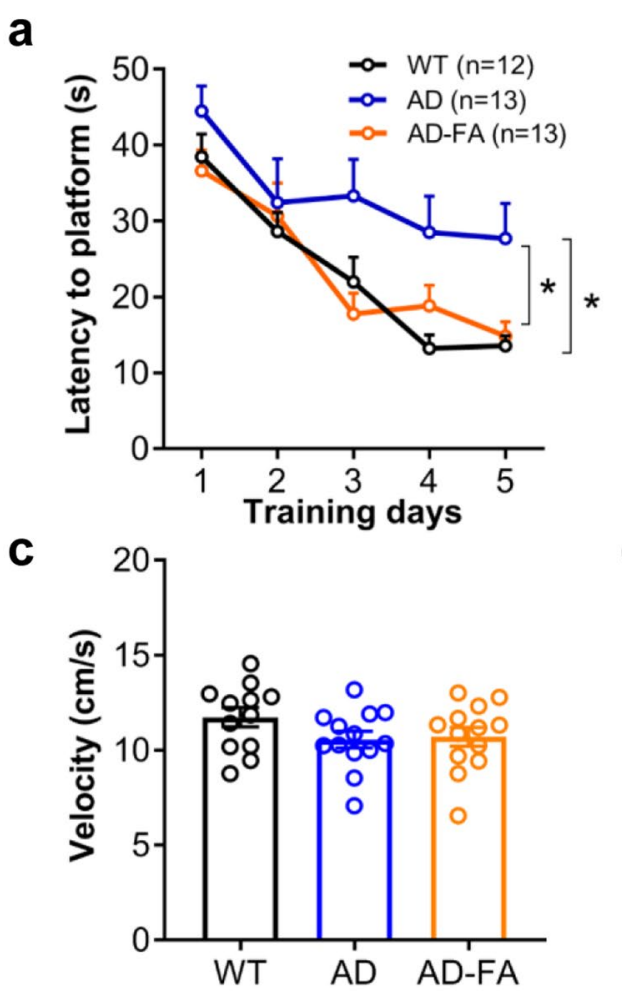

Fig. 6 FA repairs spatial memory deficit in AD mice at 7 months old. (a) Spatial learning, as indicated by latency in escaping onto a hidden platform during 5-day training, suggested that the FA treatment but not vehicle treatment prevented learning deficit in AD mice relative to WT at 7 months old. (b) Spatial memory, as indicated by the time spent in the target quadrant (s) during the probe test $24 \mathrm{~h}$ after the final training, was impaired in $\mathrm{AD}$ mice after the vehicle treat-

restoration of impaired spatial learning by the FA treatment. Probe test $24 \mathrm{~h}$ after learning suggested that AD mice with the FA treatment fully restored spatial memory deficit, which was observed in $\mathrm{AD}$ mice with the vehicle treatment (Fig. 6b, WT, $N=12$; AD or AD-FA, $N=13$; WT vs. AD, $* P=0.023$; WT $v s$. AD-FA: $P=0.972$; AD vs. AD-FA: $* P=0.011$. One-way ANOVA followed by Tukey's post hoc). These protective effects of FA on impaired spatial learning and memory were not relevant to any changes of swimming speed (Fig. 6c, d, WT, $N=12$; AD or AD-FA, $N=13$; WT $v s$. AD, $P=0.208$; WT $v$. AD-FA: $P=0.298$; AD vs. AD-FA: $P=0.972$. One-way ANOVA followed by Tukey's post hoc).

Taken together, these data strongly suggested that reduced density/diameter of hippocampal capillaries could be also necessary for initiating $A \beta$ plaque deposition and spatial memory deficit in $\mathrm{AD}$ mice at the initial stages, as indicated by FA treatment for 30 days prevented reduced density/ diameter of hippocampal capillaries and repaired spatial memory deficit completely but $\mathrm{A} \beta$ plaque deposition and aggregative microglial cells partially. b
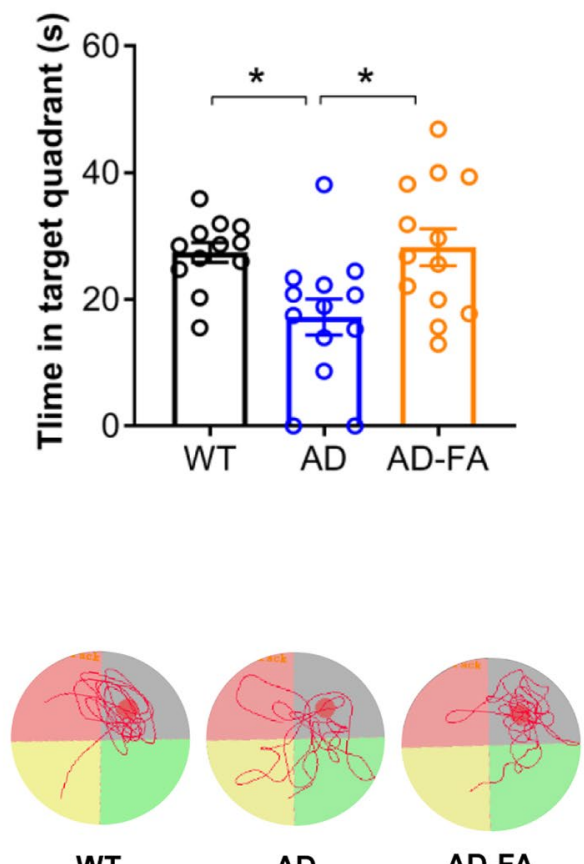

AD

AD-FA

ment. This impaired spatial memory was effectively prevented by the FA treatment, to the levels without difference from that in WT mice. (c) Mean of the swimming speed during a training trial on the first day was not different among the groups. (d) Representative tracking traces during the probe test of the Morris water maze for measuring spatial memory. Data are presented as mean \pm SEM. ${ }^{*} P<0.05$, $* * P<0.01$

\section{Discussion}

In the present study, we demonstrate that hypoperfusion of the hippocampus due to reduced density and diameter of capillaries is probably earlier than and crucial for initiating $\mathrm{A} \beta$ plaque deposition and spatial memory deficit, providing new evidence supporting a feedforward cycle of the hypoperfusion-A $\beta$ aggregation-more hypoperfusion [10] (Fig. 7) and for understanding why the prevalence of $\mathrm{AD}$ is dramatically increased following aging [1].

The earliest sign of memory decline in AD is mostly restricted to the impairment of episodic memories [34], for which the hippocampus plays a crucial role [35]. Studies on the underlying mechanisms should prompt investigation into the therapeutic control of this impairment. Previous studies have suggested that episodic memory deficit in early $\mathrm{AD}$ is attributable to ineffective encoding or failed retrieval of memory in AD patients or rodent models [72-75]. Consistent with these findings, we report that spatial learning and memory are both impaired in AD mice at 7 months old, for which reduced density and diameter of hippocampal 
Fig. 7 The schematic diagram for a feedforward cycle among capillary, $\mathrm{A} \beta$ plaque, and memory loss in AD. Decreased $\mathrm{CD} / \mathrm{CBF}$ and increased $\mathrm{A} \beta$ aggregation could form a feedforward cycle (red and green arrows), leading to memory loss and $\mathrm{A} \beta$ plaque deposition that trigger other cascades of the pathophysiology. This feedforward cycle at the initial stages could be antagonized by FA via targeting the ETRA (yellow box)

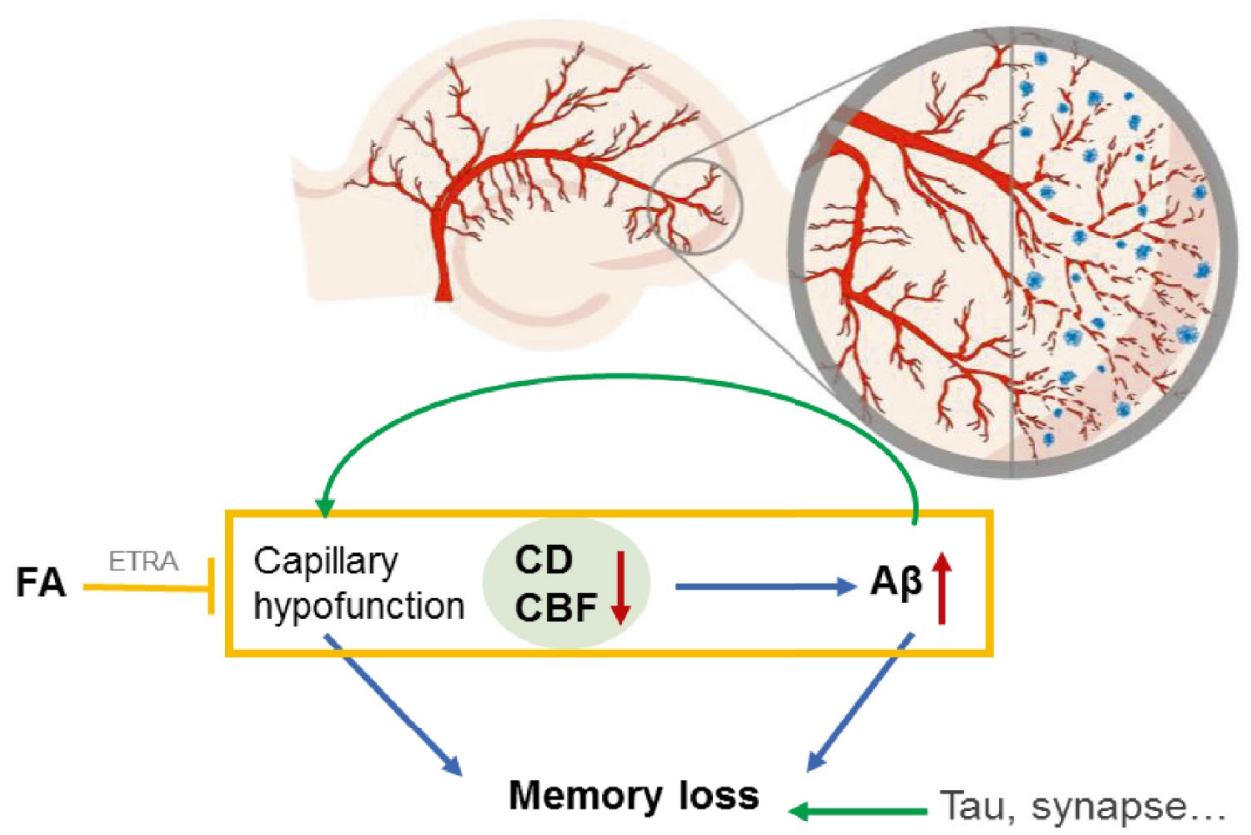

FA: Ferulic acid

CD: Capillary density CBF: Cerebral blood flow

Yellow box: Summary of our results in the paper Green arrows: Previous journal reports capillaries are the earliest events. These vascular changes would have caused a tiny hypoperfusion insult, not easily detectable in the hippocampus. We thus mimicked this situation by the injection of ET1 into the hippocampus and found that ET1 induced a small detectable hypoperfusion insult and early $\mathrm{A} \beta$ plaque deposition at 5 months old. The cause and the effect may have jointly caused the double-hits [19, 67] on the hippocampal functions of spatial learning and memory. This may have well explained why the A $\beta$-directed therapies alone have failed in halting or curing memory decline in AD patients [11, 76-78] because hypoperfusion of brain capillaries was not solved. Thus, a combination of the $A \beta$-directed therapies with those agents targeting brain capillaries could be beneficial for memory decline in $\mathrm{AD}$ at the early stages.

An alternative strategy is to prevent hypoperfusion of hippocampal capillaries as early as possible in the aging population. Our data reveal that hypoperfusion of hippocampal capillaries could be also necessary for initiating $A \beta$ plaque deposition and spatial memory deficit because FA treatment for 30 days not only prevents reduced density and diameter of brain capillaries via the ETRA but also repairs spatial memory deficit completely. Consistent with our findings, reduction in the ET-1 levels or RAGE receptor expression has been also reported contributing to the improvement of vascular function. RAGE-dependent BBB transport of circulating $A \beta$ results in the production of ET- 1 to cause decreased CBF; infusion of either RAGE-specific IgG, soluble RAGE (as a decoy receptor), or RAGE inhibitor treatment in $\mathrm{AD}$ transgenic mice causes an increase in $\mathrm{CBF}$ and reduction in brain $A \beta$ levels $[79,80]$. The RAGE blocker, FPS-ZM1, has been broadly used in various experimental paradigms in more than 75 follow-up studies. Moreover, small molecule RAGE blockers are currently understudying in the phase $2 / 3$ trial in patients with mild Alzheimer's and impaired glucose tolerance (ClinicalTrials.gov identifier NCT03980730). Thus, preventing vascular hypofunction is a promising strategy for $\mathrm{AD}$ treatment.

Furthermore, a single mechanism of action is hard to produce fully beneficial effects in AD because AD is a multifaceted disease and a combination of several drugs with multiple mechanisms of action is suggested to be essential. Here, we demonstrate that a natural compound FA can produce multiple effects on hippocampal capillaries, spatial memory, $A \beta$ plaque deposition, and aggregative microglial cells. Some earlier preclinical studies also have demonstrated the protective effects of FA or FA based hybrids in AD mouse models. In these studies, FA was found to produce actions such as reducing $A \beta$ deposition or promoting anti-inflammatory or antioxidant effects [37, $69,71,81-83]$. The present study further strengthened this idea, for which FA not only caused a reduction of $A \beta$ plaques and aggregative microglial cells but also produced protective 
effects on capillaries possibly via the ETRA, which have not been reported before. It is important to note that reduced density and diameter of hippocampal capillaries could be particularly crucial for $\mathrm{AD}$ at the earliest stages. FA intervention later than these stages could be ineffective because $A \beta$ plaque deposition and aggregative microglial cells could have caused other cascades of the pathophysiology of AD [2-10]. Moreover, our RNA sequencing analysis suggested distinct profiles of gene expression for AD mice with the 30-day FA or vehicle treatment relative to WT mice, further supporting multiple effects of $\mathrm{FA}$ on brain capillaries and $\mathrm{A} \beta$ plaque deposition, and other possible actions (Supplementary Fig. 7). Notably, a recent multicenter, randomized, double-blinded clinical study has investigated the effects of Feru-guard that contained FA for treating mild cognition impairment (MCI) patients aged 65 to 85 years old and the results strongly support the opinion that FA is beneficial for MCI [84], a possible early stage of AD.

It is urgent to find disease-modifying therapy to halt or cure the progressive decline of memory and other cognitive functions in AD. Here, our data provide novel evidence supporting that FA supplement taking as early as possible against the ET1-mediated ETRA activation on hippocampal capillaries is potentially beneficial for memory decline in $\mathrm{AD}$ at the earliest stages.

Supplementary Information The online version contains supplementary material available at https://doi.org/10.1007/s13311-021-01024-7.

Acknowledgements We thank J.F. Li for the synthesis of FA-biotin, and all members of Lin Xu's laboratory for their supports.

Required Author Forms Disclosure forms provided by the authors are available with the online version of this article.

Funding This work was supported by the Natural Science Foundation of China (32071029 to Q.X.Z., and 31861143037 to L.X. and G.R.-L.), and Strategic Priority Research Program of the Chinese Academy of Sciences (XDB32020000 to L.X.), and External Cooperation Program BIC of the Chinese Academy of Sciences (GJHZ1549 to G.R.L.), and Youth Innovation Promotion Association of the Chinese Academy of Sciences (2013250 to Q.X.Z.), and Department of Science and Technology Program of Yunnan Province (2016HA002 to L.X.).

\section{Declarations}

Conflict of interest Some authors are listed as the inventors of a CN Patent (application no. 202010395578.7). The other authors declare that they have no competing interests.

Open Access This article is licensed under a Creative Commons Attribution 4.0 International License, which permits use, sharing, adaptation, distribution and reproduction in any medium or format, as long as you give appropriate credit to the original author(s) and the source, provide a link to the Creative Commons licence, and indicate if changes were made. The images or other third party material in this article are included in the article's Creative Commons licence, unless indicated otherwise in a credit line to the material. If material is not included in the article's Creative Commons licence and your intended use is not permitted by statutory regulation or exceeds the permitted use, you will need to obtain permission directly from the copyright holder. To view a copy of this licence, visit http://creativecommons.org/licenses/by/4.0/.

\section{References}

1. Nativio R, Donahue G, Berson A, Lan Y, Amlie-Wolf A, Tuzer F, et al. Dysregulation of the epigenetic landscape of normal aging in Alzheimer's disease. Nat Neurosci 2018;21(4):497-505.

2. Glenner GG, Wong CW. Alzheimer's disease: initial report of the purification and characterization of a novel cerebrovascular amyloid protein. Biochem Biophys Res Commun 1984;120(3):885-90.

3. Beyreuther K, Masters CL. Amyloid precursor protein (APP) and beta A4 amyloid in the etiology of Alzheimer's disease: precursorproduct relationships in the derangement of neuronal function. Brain pathology (Zurich, Switzerland) 1991;1(4):241-51.

4. Hardy JA, Higgins GA. Alzheimer's disease: the amyloid cascade hypothesis. Science 1992;256(5054):184-5.

5. Duyckaerts C, Delatour B, Potier MC. Classification and basic pathology of Alzheimer disease. Acta Neuropathol 2009;118(1):5-36.

6. De Strooper B, Karran E. The Cellular Phase of Alzheimer's Disease. Cell 2016;164(4):603-15.

7. Selkoe DJ, Hardy J. The amyloid hypothesis of Alzheimer's disease at 25 years. EMBO Mol Med 2016;8(6):595-608.

8. Jack CR, Jr., Bennett DA, Blennow K, Carrillo MC, Dunn B, Haeberlein SB, et al. NIA-AA Research Framework: Toward a biological definition of Alzheimer's disease. Alzheimers Dement 2018;14(4):535-62.

9. Sun Q, Xie N, Tang B, Li R, Shen Y. Alzheimer's Disease: From Genetic Variants to the Distinct Pathological Mechanisms. Front Mol Neurosci 2017;10:319.

10. Wang J, Gu BJ, Masters CL, Wang YJ. A systemic view of Alzheimer disease - insights from amyloid- $\beta$ metabolism beyond the brain. Nat Rev Neurol 2017;13(10):612-23.

11. Long JM, Holtzman DM. Alzheimer Disease: An Update on Pathobiology and Treatment Strategies. Cell 2019;179(2):312-39.

12. Johnson KA, Jones K, Holman BL, Becker JA, Spiers PA, Satlin A, et al. Preclinical prediction of Alzheimer's disease using SPECT. Neurology 1998;50(6):1563-71.

13. Asllani I, Habeck C, Scarmeas N, Borogovac A, Brown TR, Stern Y. Multivariate and univariate analysis of continuous arterial spin labeling perfusion MRI in Alzheimer's disease. J Cereb Blood Flow Metab 2008;28(4):725-36.

14. Austin BP, Nair VA, Meier TB, Xu G, Rowley HA, Carlsson CM, et al. Effects of hypoperfusion in Alzheimer's disease. J Alzheimers Dis 2011;26(Suppl 3):123-33.

15. Mazza M, Marano G, Traversi G, Bria P, Mazza S. Primary cerebral blood flow deficiency and Alzheimer's disease: shadows and lights. J Alzheimers Dis 2011;23(3):375-89.

16. Baloyannis SJ, Baloyannis IS. The vascular factor in Alzheimer's disease: a study in Golgi technique and electron microscopy. J Neurol Sci 2012;322(1-2):117-21.

17. Love S, Miners JS. Cerebrovascular disease in ageing and Alzheimer's disease. Acta Neuropathol 2016;131(5):645-58.

18. Iturria-Medina Y, Sotero RC, Toussaint PJ, Mateos-Pérez JM, Evans AC. Early role of vascular dysregulation on late-onset Alzheimer's disease based on multifactorial data-driven analysis. Nat Commun 2016;7:11934.

19. Kisler K, Nelson AR, Montagne A, Zlokovic BV. Cerebral blood flow regulation and neurovascular dysfunction in Alzheimer disease. Nat Rev Neurosci 2017;18(7):419-34.

20. Jin WS, Bu XL, Wang YR, Li L, Li WW, Liu YH, et al. Reduced Cardiovascular Functions in Patients with Alzheimer's Disease. J Alzheimers Dis 2017;58(3):919-25.

21. Nielsen RB, Egefjord L, Angleys H, Mouridsen K, Gej1 M, Møller A, et al. Capillary dysfunction is associated with symptom 
severity and neurodegeneration in Alzheimer's disease. Alzheimers Dement 2017;13(10):1143-53.

22. Hecht M, Krämer LM, von Arnim CAF, Otto M, Thal DR. Capillary cerebral amyloid angiopathy in Alzheimer's disease: association with allocortical/hippocampal microinfarcts and cognitive decline. Acta Neuropathol 2018;135(5):681-94.

23. De Jong GI, Farkas E, Stienstra CM, Plass JR, Keijser JN, de la Torre JC, et al. Cerebral hypoperfusion yields capillary damage in the hippocampal CA1 area that correlates with spatial memory impairment. Neuroscience 1999;91(1):203-10.

24. Cruz Hernández JC, Bracko O, Kersbergen CJ, Muse V, HaftJavaherian M, Berg M, et al. Neutrophil adhesion in brain capillaries reduces cortical blood flow and impairs memory function in Alzheimer's disease mouse models. Nat Neurosci 2019;22(3):413-20.

25. Xiaochuan, Zhang, Xianzhen, Yin, Jinging, Zhang, et al. Highresolution mapping of brain vasculature and its impairment in the hippocampus of Alzheimer's disease mice. National Science Review 2019;v.6(06):167-82.

26. Nortley R, Korte N, Izquierdo P, Hirunpattarasilp C, Mishra A, Jaunmuktane $\mathrm{Z}$, et al. Amyloid $\beta$ oligomers constrict human capillaries in Alzheimer's disease via signaling to pericytes. Science 2019;365(6450).

27. Suo Z, Humphrey J, Kundtz A, Sethi F, Placzek A, Crawford F, et al. Soluble Alzheimers beta-amyloid constricts the cerebral vasculature in vivo. Neurosci Lett 1998;257(2):77-80.

28. Dietrich HH, Xiang C, Han BH, Zipfel GJ, Holtzman DM. Soluble amyloid-beta, effect on cerebral arteriolar regulation and vascular cells. Mol Neurodegener 2010;5:15.

29. Sun X, He G, Qing H, Zhou W, Dobie F, Cai F, et al. Hypoxia facilitates Alzheimer's disease pathogenesis by up-regulating BACE1 gene expression. Proc Natl Acad Sci USA 2006;103(49):18727-32.

30. Zhang X, Le W. Pathological role of hypoxia in Alzheimer's disease. Exp Neurol 2010;223(2):299-303.

31. Sagare AP, Bell RD, Zlokovic BV. Neurovascular dysfunction and faulty amyloid $\beta$-peptide clearance in Alzheimer disease. Cold Spring Harb Perspect Med 2012;2(10).

32. Marques F, Sousa JC, Sousa N, Palha JA. Blood-brain-barriers in aging and in Alzheimer's disease. Mol Neurodegener 2013;8:38.

33. Baloyannis SJ. Brain capillaries in Alzheimer's disease. Hell J Nucl Med 2015;18 Suppl 1:152.

34. Selkoe DJ. Alzheimer's disease is a synaptic failure. Science 2002;298(5594):789-91.

35. Morris RG, Garrud P, Rawlins JN, O'Keefe J. Place navigation impaired in rats with hippocampal lesions. Nature 1982;297(5868):681-3.

36. Iadecola C, Gottesman RF. Cerebrovascular Alterations in Alzheimer Disease. Circ Res 2018;123(4):406-8.

37. Sgarbossa A, Giacomazza D, di Carlo M. Ferulic Acid: A Hope for Alzheimer's Disease Therapy from Plants. Nutrients 2015;7(7):5764-82.

38. Hamaguchi T, Ono K, Murase A, Yamada M. Phenolic compounds prevent Alzheimer's pathology through different effects on the amyloid-beta aggregation pathway. Am J Pathol 2009;175(6):2557-65.

39. Vorhees CV, Williams MT. Morris water maze: procedures for assessing spatial and related forms of learning and memory. Nat Protoc 2006;1(2):848-58.

40. Li J, Chai A, Wang L, Ma Y, Wu Z, Yu H, et al. Synaptic P-Rex1 signaling regulates hippocampal long-term depression and autism-like social behavior. Proc Natl Acad Sci USA 2015;112(50):E6964-72.

41. Li HB, Mao RR, Zhang JC, Yang Y, Cao J, Xu L. Antistress effect of TRPV1 channel on synaptic plasticity and spatial memory. Biol Psychiatry 2008;64(4):286-92.

42. Yang Y, Cao J, Xiong W, Zhang J, Zhou Q, Wei H, et al. Both stress experience and age determine the impairment or enhancement effect of stress on spatial memory retrieval. Journal Endocrinol 2003;178(1):45-54.
43. Winkler EA, Nishida Y, Sagare AP, Rege SV, Bell RD, Perlmutter D, et al. GLUT1 reductions exacerbate Alzheimer's disease vasculo-neuronal dysfunction and degeneration. Nat Neurosci 2015;18(4):521-30.

44. Ridder DA, Wenzel J, Müller K, Töllner K, Tong XK, Assmann JC, et al. Brain endothelial TAK1 and NEMO safeguard the neurovascular unit. J Exp Med 2015;212(10):1529-49.

45. Sharkey J, Butcher SP. Characterisation of an experimental model of stroke produced by intracerebral microinjection of endothelin-1 adjacent to the rat middle cerebral artery. J Neurosci Methods 1995;60(1-2):125-31.

46. Zhou H, Xiong GJ, Jing L, Song NN, Pu DL, Tang X, et al. The interhemispheric CA1 circuit governs rapid generalisation but not fear memory. Nat Commun 2017;8(1):2190.

47. Jiang L, Mao R, Tong J, Li J, Chai A, Zhou Q, et al. Inhibition of Rac1 activity in the hippocampus impaired extinction of contextual fear. Neuropharmacology. 2016;109:216-22.

48. Sun R, Chen X, Yin CY, Qi L, Lau PM, Han H, et al. Correlative light and electron microscopy for complex cellular structures on PDMS substrates with coded micro-patterns. Lab Chip 2018;18(24):3840-8.

49. Dunn AK. Laser speckle contrast imaging of cerebral blood flow. Ann Biomed Eng 2012;40(2):367-77.

50. Yu CL, Zhou H, Chai AP, Yang YX, Mao RR, Xu L. Wholescale neurobehavioral assessments of photothrombotic ischemia in freely moving mice. J Neurosci Methods 2015;239:100-7.

51. Watson BD, Dietrich WD, Busto R, Wachtel MS, Ginsberg MD. Induction of reproducible brain infarction by photochemically initiated thrombosis. Ann Neurol 1985;17(5):497-504.

52. Roychowdhury S, Koozekanani DD, Parhi KK. Iterative Vessel Segmentation of Fundus Images. IEEE Transactions On Biomedical Engineering 2015;62(7):1738-49.

53. Dong YB, Li MJ, Sun Y. Research on Threshold Segmentation Algorithms. Adv Mater Res 2013;860-863:2888-91.

54. Waterhouse A, Bertoni M, Bienert S, Studer G, Tauriello G, Gumienny R, et al. SWISS-MODEL: homology modelling of protein structures and complexes. Nucleic Acids Res 2018;46(W1):W296-w303.

55. Camacho C, Coulouris G, Avagyan V, Ma N, Papadopoulos J, Bealer K, et al. BLAST+: architecture and applications. BMC bioinformatics 2009;10:421.

56. Remmert M, Biegert A, Hauser A, Söding J. HHblits: lightningfast iterative protein sequence searching by HMM-HMM alignment. Nature methods 2011;9(2):173-5.

57. Trott O, Olson AJ. AutoDock Vina: improving the speed and accuracy of docking with a new scoring function, efficient optimization, and multithreading. J Comput Chem 2010;31(2):455-61.

58. Jiang H, Lei R, Ding SW, Zhu S. Skewer: a fast and accurate adapter trimmer for next-generation sequencing paired-end reads. BMC Bioinformatics 2014;15:182.

59. Kim D, Langmead B, Salzberg SL. HISAT: a fast spliced aligner with low memory requirements. Nature Methods 2015;12(4):357-60.

60. Pertea M, Kim D, Pertea GM, Leek JT, Salzberg SL. Transcriptlevel expression analysis of RNA-seq experiments with HISAT, StringTie and Ballgown. Nat Protoc 2016;11(9):1650-67.

61. Li H, Handsaker B, Wysoker A, Fennell T, Ruan J, Homer N, et al. The Sequence Alignment/Map format and SAMtools. Bioinformatics (Oxford, England) 2009;25(16):2078-9.

62. Pertea M, Pertea GM, Antonescu CM, Chang TC, Mendell JT, Salzberg SL. StringTie enables improved reconstruction of a transcriptome from RNA-seq reads. Nat Biotechnol 2015;33(3):290-5.

63. Frazee AC, Pertea G, Jaffe AE, Langmead B, Salzberg SL, Leek JT. Ballgown bridges the gap between transcriptome assembly and expression analysis. Nat Biotechnol 2015;33(3):243-6.

64. Kinsella RJ, Kähäri A, Haider S, Zamora J, Proctor G, Spudich G, et al. Ensembl BioMarts: a hub for data retrieval across taxonomic space. Database: The Journal of Biological Databases and Curation 2011;2011:bar030. 
65. Ritchie ME, Phipson B, Wu D, Hu Y, Law CW, Shi W, et al. limma powers differential expression analyses for RNA-sequencing and microarray studies. Nucleic Acids Res 2015;43(7):e47.

66. Nelson PT, Alafuzoff I, Bigio EH, Bouras C, Braak H, Cairns NJ, et al. Correlation of Alzheimer disease neuropathologic changes with cognitive status: a review of the literature. J Neuropathol Exp Neurol 2012;71(5):362-81.

67. Østergaard L, Aamand R, Gutiérrez-Jiménez E, Ho YC, Blicher JU, Madsen SM, et al. The capillary dysfunction hypothesis of Alzheimer's disease. Neurobiol Aging 2013;34(4):1018-31.

68. Hainsworth AH, Brittain JF, Khatun H. Pre-clinical models of human cerebral small vessel disease: utility for clinical application. J Neurol Sci 2012;322(1-2):237-40.

69. Mori T, Koyama N, Guillot-Sestier MV, Tan J, Town T. Ferulic acid is a nutraceutical $\beta$-secretase modulator that improves behavioral impairment and alzheimer-like pathology in transgenic mice. PLoS One 2013;8(2):e55774.

70. Lazic D, Sagare AP, Nikolakopoulou AM, Griffin JH, Vassar R, Zlokovic BV. 3K3A-activated protein $\mathrm{C}$ blocks amyloidogenic BACE1 pathway and improves functional outcome in mice. J Exp Med 2019;216(2):279-93.

71. Tripathi A, Choubey PK, Sharma P, Seth A, Saraf P, Shrivastava SK. Design, synthesis, and biological evaluation of ferulic acid based 1,3,4-oxadiazole hybrids as multifunctional therapeutics for the treatment of Alzheimer's disease. Bioorg Chem 2020;95:103506.

72. Granholm E, Butters N. Associative encoding and retrieval in Alzheimer's and Huntington's disease. Brain Cogn 1988;7(3):335-47.

73. Hodges JR, Salmon DP, Butters N. Differential impairment of semantic and episodic memory in Alzheimer's and Huntington's diseases: a controlled prospective study. J Neurol Neurosurg Psychiatry 1990;53(12):1089-95.

74. Weintraub S, Wicklund AH, Salmon DP. The neuropsychological profile of Alzheimer disease. Cold Spring Harb Perspect Med 2012;2(4):a006171.

75. Roy DS, Arons A, Mitchell TI, Pignatelli M, Ryan TJ, Tonegawa $\mathrm{S}$. Memory retrieval by activating engram cells in mouse models of early Alzheimer's disease. Nature 2016;531(7595):508-12.
76. Mangialasche F, Solomon A, Winblad B, Mecocci P, Kivipelto M. Alzheimer's disease: clinical trials and drug development. Lancet Neurol 2010;9(7):702-16.

77. Huang Y, Mucke L. Alzheimer mechanisms and therapeutic strategies. Cell 2012;148(6):1204-22.

78. Panza F, Lozupone M, Logroscino G, Imbimbo BP. A critical appraisal of amyloid- $\beta$-targeting therapies for Alzheimer disease. Nat Rev Neurol 2019;15(2):73-88.

79. Deane R, Du Yan S, Submamaryan RK, LaRue B, Jovanovic S, Hogg E, et al. RAGE mediates amyloid-beta peptide transport across the blood-brain barrier and accumulation in brain. Nat Med 2003;9(7):907-13.

80. Deane R, Singh I, Sagare AP, Bell RD, Ross NT, LaRue B, et al. A multimodal RAGE-specific inhibitor reduces amyloid $\beta$-mediated brain disorder in a mouse model of Alzheimer disease. J Clin Invest 2012;122(4):1377-92.

81. Yan JJ, Jung JS, Kim TK, Hasan A, Hong CW, Nam JS, et al. Protective effects of ferulic acid in amyloid precursor protein plus presenilin-1 transgenic mouse model of Alzheimer disease. Biol Pharm Bull 2013;36(1):140-3.

82. Jung JS, Yan JJ, Li HM, Sultan MT, Yu J, Lee HS, et al. Protective effects of a dimeric derivative of ferulic acid in animal models of Alzheimer's disease. Eur J Pharmacol 2016;782:30-4.

83. Mori T, Koyama N, Tan J, Segawa T, Maeda M, Town T. Combined treatment with the phenolics (-)-epigallocatechin-3-gallate and ferulic acid improves cognition and reduces Alzheimer-like pathology in mice. J Biol Chem 2019;294(8):2714-31.

84. Kudoh C, Hori T, Yasaki S, Ubagai R, Tabira T. Effects of Ferulic Acid and Angelica archangelica Extract (Feru-guard(®)) on Mild Cognitive Impairment: A Multicenter, Randomized, DoubleBlind, Placebo-Controlled Prospective Trial. J Alzheimers Dis Rep 2020;4(1):393-8.

Publisher's Note Springer Nature remains neutral with regard to jurisdictional claims in published maps and institutional affiliations.

\section{Authors and Affiliations}

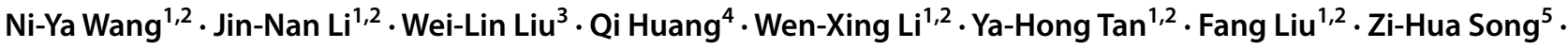 Meng-Yue Wang ${ }^{6} \cdot$ Ning Xie $^{6} \cdot$ Rong-Rong Mao $^{1,7} \cdot$ Ping Gan $^{1,7} \cdot$ Yu-Qiang Ding ${ }^{8} \cdot$ Zhi Zhang $^{5} \cdot$ Bao-Ci Shan ${ }^{4}$. Li-Dian Chen ${ }^{3} \cdot$ Qi-Xin Zhou ${ }^{1,2} \cdot$ Lin Xu $\mathbf{u}^{1,2,9,10}$}

1 CAS Key Laboratory of Animal Models and Human Disease Mechanisms, and KIZ-SU Joint Laboratory of Animal Model and Drug Development, and Laboratory of Learning and Memory, Kunming Institute of Zoology, the Chinese Academy of Sciences, Kunming 650223, China

2 Kunming College of Life Sciences, University of the Chinese Academy of Sciences, Kunming 650223, China

3 The Academy of Rehabilitation Industry, Fujian University of Traditional Chinese Medicine, Fuzhou 350122, China

4 Key Laboratory of Nuclear Analysis Techniques, Institute of High Energy Physics, the Chinese Academy of Sciences, Beijing 100049, China

5 CAS Key Laboratory of Brain Function and Disease, Hefei National Laboratory for Physical Sciences At the Microscale, University of Science and Technology of China,

Hefei 230027, China
6 State Key Laboratory of Innovative Natural Drugs and Traditional Chinese Medicine Injections, Qingfeng Pharmaceutical Corporations, Ganzhou 341000, China

7 Kunming Medical University, Kunming 650500, China

8 State Key Laboratory of Medical Neurobiology and MOE Frontiers Centre for Brain Science, Institutes of Brain Science, Fudan University, Shanghai 200032, China

9 Mental Health Institute, the Second Xiangya Hospital of Central South University, Changsha 410008, China

10 CAS Centre for Excellence in Brain Science and Intelligent Technology, Shanghai 200031, China 